\title{
Polarimetric X-band weather radar measurements in the tropics: radome and rain attenuation correction
}

\author{
M. Schneebeli ${ }^{1,2, *}$, J. Sakuragi ${ }^{1}$, T. Biscaro ${ }^{1}$, C. F. Angelis ${ }^{1}$, I. Carvalho da Costa $^{1}$, C. Morales ${ }^{3}$, L. Baldini ${ }^{4}$, and \\ L. A. T. Machado ${ }^{1}$ \\ ${ }^{1}$ Instituto Nacional de Pesquisas Espaciais (INPE), Centro de Previsão de Tempo e Estudos Climáticos (CPTEC), \\ Cachoeira Paulista, Brazil \\ ${ }^{2}$ École Polytechnique Fédérale de Lausanne (EPFL), School of Architecture, Civil and Environmental Engineering (ENAC), \\ Lausanne, Switzerland \\ ${ }^{3}$ Instituto de Astronomia, Geofisica e Ciéncias Atmosféricas, Universidade de São Paulo, São Paulo, Brazil \\ ${ }^{4}$ Instituto di Scienze dell' Atmosfera e del Clima (CNR), Rome, Italy \\ *now at: MeteoSwiss, Radar and Satellites, Locarno, Switzerland
}

Correspondence to: M. Schneebeli (marc.schneebeli@epfl.ch)

Received: 23 January 2012 - Published in Atmos. Meas. Tech. Discuss.: 17 February 2012

Revised: 14 June 2012 - Accepted: 24 July 2012 - Published: 10 September 2012

\begin{abstract}
A polarimetric X-band radar has been deployed during one month (April 2011) for a field campaign in Fortaleza, Brazil, together with three additional laser disdrometers. The disdrometers are capable of measuring the raindrop size distributions (DSDs), hence making it possible to forward-model theoretical polarimetric X-band radar observables at the point where the instruments are located. This setup allows to thoroughly test the accuracy of the X-band radar measurements as well as the algorithms that are used to correct the radar data for radome and rain attenuation. For the campaign in Fortaleza it was found that radome attenuation dominantly affects the measurements. With an algorithm that is based on the self-consistency of the polarimetric observables, the radome induced reflectivity offset was estimated. Offset corrected measurements were then further corrected for rain attenuation with two different schemes. The performance of the post-processing steps was analyzed by comparing the data with disdrometer-inferred polarimetric variables that were measured at a distance of $20 \mathrm{~km}$ from the radar. Radome attenuation reached values up to $14 \mathrm{~dB}$ which was found to be consistent with an empirical radome attenuation vs. rain intensity relation that was previously developed for the same radar type. In contrast to previous work, our results suggest that radome attenuation should be estimated individually for every view direction of the radar in order to obtain homogenous reflectivity fields.
\end{abstract}

\section{Introduction}

The usage of conventional single polarization X-band weather radars was limited to target applications on small rural or urban basins, also serving as a gap filler of conventional weather radar networks (e.g. Delrieu et al., 1999). When dual-polarization X-band radars became available in the last decade (Zrnic and Ryzhkov, 1999) that had the capability of correcting rain attenuation in a reliable manner (Testud et al., 2000; Anagnostou et al., 2004; Gorgucci et al., 2006), these instruments became a popular tool for various applications related to the measurement of precipitation at small to medium scales $(\approx 1-50 \mathrm{~km})$. This culminated in the proposal of networking operations with the potential to cover wide areas (like e.g. the CASA project; McLaughlin et al., 2009; Wang and Chandrasekar, 2010)

The fact that X-band radars are small and therefore easy to deploy makes them attractive for use in field campaigns, hence numerous field studies have made use of X-band radar measurements up to now. The HMT campaign (Matrosov, 2010) as well as the campaign described in Schneebeli et al. (2010) focused on measurements of snowfall, while others aimed at better understanding the microphysics of precipitation (Berne et al., 2005; Anagnostou et al., 2006).

In April 2011, the first field campaign of the CHUVA project took place in Fortaleza, on the northeastern coast 
of Brazil. CHUVA (being the Portuguese word for rain, the acronym stands for Cloud processes of tHe main precipitation systems in Brazil: A contribUtion to cloud resolVing modeling and to the GPM (GlobAl Precipitation Measurement)) aims at resolving microphysical processes that occur in tropical clouds in order to better understand formation of severe precipitation. The set-up of remote sensors consisted of an X-band polarimetric weather radar (see e.g. Bechini et al., 2010), a $24 \mathrm{GHz}$ vertically pointing Micro Rain Radar (MRR; Peters et al., 2002), a Raymetrics Raman lidar, and a Radiometrics passive microwave temperature and humidity profiler (Ware et al., 2003), and was complemented by a wealth of ground-based (disdrometers, rain gauges, anemometric tower) and air-borne (triangle of radiosondes launched every six hours) in-situ sensors. With this sensor assembly deployed in the tropics, a detailed assessment of processes governing the formation of clouds and precipitation became feasible. The focus of the first campaign in Fortaleza was on the detection of warm clouds, i.e. clouds that do not exceed the melting layer and therefore form precipitation without the influence of the ice phase. Rain events originating from warm clouds can be very hazardous, but detection with traditional satellite-based precipitation estimation schemes is difficult due to the lack of the ice phase of these clouds and therefore remains an unresolved problem. It is expected that the results from the Fortaleza campaign will improve the understanding of warm rain events and eventually lead to better rain estimates over land. Following the campaign in Fortaleza, the whole sensor assembly was moved to Belém. The focus of the Belém campaign, which took place in June 2011, was on tropical squall-lines and their associated processes that form intense tropical precipitation. In order to make the radar data useful for any quantitative analysis and for using it in microphysical studies, several post-processing steps were necessary, such as radome and rain attenuation correction, differential reflectivity correction, estimation of the specific differential phase and hydrometeor identification. Several algorithms capable of tackling these tasks were implemented. The ZPHI algorithm from Testud et al. (2000) was used for correcting the reflectivity $Z_{\mathrm{h}}$ and differential reflectivity $Z_{\mathrm{dr}}$ for attenuation that is due to the rain medium. The algorithm from Hubbert and Bringi (1995) was used for the estimation of the accumulated and specific differential propagation phase shift $\Phi_{\mathrm{dp}}$ and $K_{\mathrm{dp}}$, respectively. Finally, the extended Kalman filter (EKF) algorithm from Schneebeli and Berne (2012) was used for rain attenuation correction of $Z_{\mathrm{h}}$ and $Z_{\mathrm{dr}}$ (similar to ZPHI), radome attenuation correction of $Z_{\mathrm{h}}$ as well as for the estimation of $K_{\mathrm{dp}}$ and $\Phi_{\mathrm{dp}}$. All these methods were implemented and tested on the first available data sets from the Fortaleza campaign. Raindrop size distributions (DSDs) provided by the disdrometers were used to forwardmodel the radar polarimetric variables by employing the Tmatrix method (Mishchenko and Travis, 1998). The results of this modeling approach are further being used for the correct adaptation of existing attenuation correction schemes to the conditions at the site in Brazil. The details of these algorithms will be described and it will also be shown how they perform by comparing radar measurements with the polarimetric moments inferred from DSD measurements of a OTT Parsivel disdrometer located at a distance of $20 \mathrm{~km}$ from the radar.

This article is organized as follows: in Sect. 2, specifications of the radar, the disdrometer and the measurements associated with these instruments are described. In Sect. 3, it is shown how polarimetric moments are calculated from the disdrometer measurements. These simulations are then used in Sects. 4 and 5, where the attenuation correction with the ZPHI algorithm is described and the EKF method is introduced, respectively. Methods for correcting $Z_{\mathrm{dr}}$ are provided in Sect. 6. Finally, the results for the radome and rain attenuation correction and the data processing with the EKF and ZPHI methods are given in Sect. 7 and conclusions are drawn in Sect. 8.

\section{Campaign set-up and instruments}

\subsection{The campaign in Fortaleza}

Fortaleza $\left(3.43^{\circ} \mathrm{S}, 38.30^{\circ} \mathrm{W}\right)$ is located at the northern coast of Brazil and counts around 2.5 million inhabitants. The radar was installed on a $5 \mathrm{~m}$ high temporary tower that was erected in a neighboring suburb called Eusebio. Within the city of Fortaleza, a measurement site (called Defesa civil) was established that was equipped with a Parsivel disdrometer, the MRR and the additional ground based instruments. The distance between the radar and the measurement site in the city was $20 \mathrm{~km}$. Two additional Parsivels were set at different locations, one at the Federal University of Céara (UECE) which was also located at a distance of $20 \mathrm{~km}$ from the radar, the other one was deployed in the suburb Caucaia at a distance of around $30 \mathrm{~km}$ from the radar. On average, Fortaleza receives $350 \mathrm{~mm}$ of rain during April and the daily mean temperature is $27^{\circ} \mathrm{C}$. During the time of the campaign, a slightly higher rain accumulation of $404 \mathrm{~mm}$ was reached, which consisted of 7 major convective rain events that exceeded intensities of $100 \mathrm{~mm} \mathrm{~h}^{-1}$. Around 11 light rain events additionally contributed to the total rain amount. The major rain events lasted around $4 \mathrm{~h}$ on average while the light rain events had shorter durations of around 1-2h. In Fig. 1, the rain rate time series of a convective rain event that took place on 12 April 2011 is plotted. The rain rates were measured with the three mentioned Parsivel disdrometers and the data collected during this event will be extensively used in the upcoming analysis.

\subsection{Radar specifications and scanning strategy}

A mobile $9.345 \mathrm{GHz}$ dual-polarization radar, manufactured by Gematronik, Germany, was employed. Its main characteristics are summarized in Table 1. 
Table 1. Specifications of the Gematronik radar.

\begin{tabular}{|c|c|}
\hline Transmitter & Magnetron delivering $35 \mathrm{~kW}$ per channel. \\
\hline Polarization & Simultaneous horizontal and vertical transmission. \\
\hline Pulses & $\begin{array}{l}\text { Staggered PRF (pulse repetition frequency) mode with PRFs of } 1200 \mathrm{~Hz} \text { and } \\
1500 \mathrm{~Hz} \text { as well as ordinary pulse pair mode (PRF: } 1500 \mathrm{~Hz}) \text {; pulse length of } \\
0.5 \mu \mathrm{s}(150 \mathrm{~m} \text { resolution). }\end{array}$ \\
\hline Antenna & $1.8 \mathrm{~m}$ diameter, $1.3^{\circ} 3-\mathrm{dB}$ beam width. \\
\hline Antenna gain & $43 \mathrm{~dB}$ \\
\hline Operating frequency & $9.375 \mathrm{GHz}$ \\
\hline Scanning protocol & Combination of RHI and volume scans. See the Sect. 2.2 for details. \\
\hline Pulse sampling & $\begin{array}{l}\text { PPI scans: } 68 \text { samples per ray @ } 1^{\circ} \text { angular resolution. RHI scans: } 150 \text { samples } \\
\text { per ray @ } 0.5^{\circ} \text { angular resolution. }\end{array}$ \\
\hline Positioning & Sun tracking method. \\
\hline
\end{tabular}

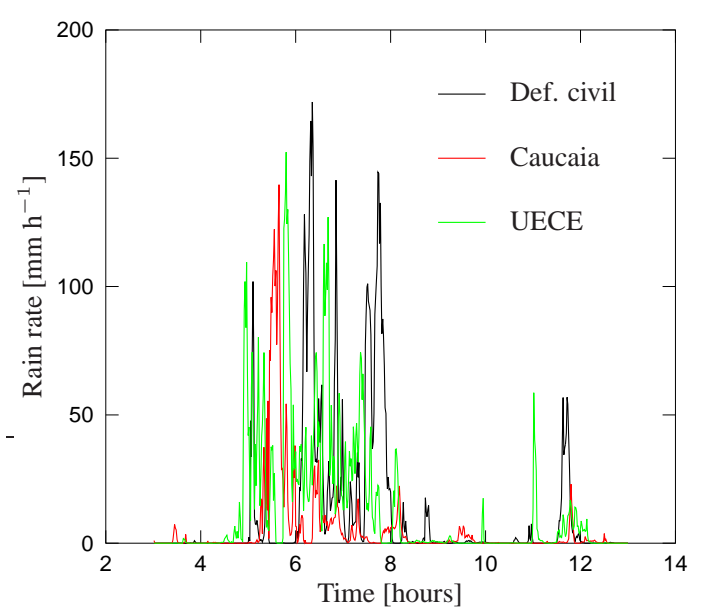

Fig. 1. Rain rate time series of a convective rain event measured on 12 April 2011. The measurement were taken with three Parsivel disdrometers that were deployed within an area of $10 \mathrm{~km}^{2}$.

The radar provides the standard polarimetric observables: reflectivity in horizontal polarization $\left(Z_{\mathrm{h}}[\mathrm{dBZ}]\right)$, differential reflectivity $\left(Z_{\mathrm{dr}}[\mathrm{dB}]\right)$, differential phase shift $\left(\Psi_{\mathrm{dp}}\left[^{\circ}\right]\right)$, specific differential phase shift $\left(K_{\mathrm{dp}}\left[{ }^{\circ} \mathrm{km}^{-1}\right]\right)$, copolar correlation coefficient $\left(\rho_{\mathrm{hv}}[-]\right)$, as well as the Doppler variables velocity $\left(v_{\mathrm{D}}\left[\mathrm{m} \mathrm{s}^{-1}\right]\right)$ and velocity spectrum width $\left(\sigma_{\mathrm{v}_{\mathrm{D}}}\right.$ $\left.\left[\mathrm{ms}^{-1}\right]\right)$. An example of one range-height indicator (RHI) scan of the radar's polarimetric variables is shown in Fig. 2.

The strategy for the Fortaleza campaign includes a $Z_{\mathrm{dr}}$ calibration scan $\left(89^{\circ}\right.$ elevation with high speed; $89^{\circ}$ instead of $90^{\circ}$ elevation chosen due to a limitation in the radar control software), two RHI scans with high angular resolution $\left(0.5^{\circ}\right)$, and high sampling frequency (150 independent samples per ray). One RHI was directed towards the heavily equipped ground site located at $20 \mathrm{~km}$ distance from the radar where also the Parsivel disdrometer is located that will later be used for evaluating the radar's polarimetric moments. The other
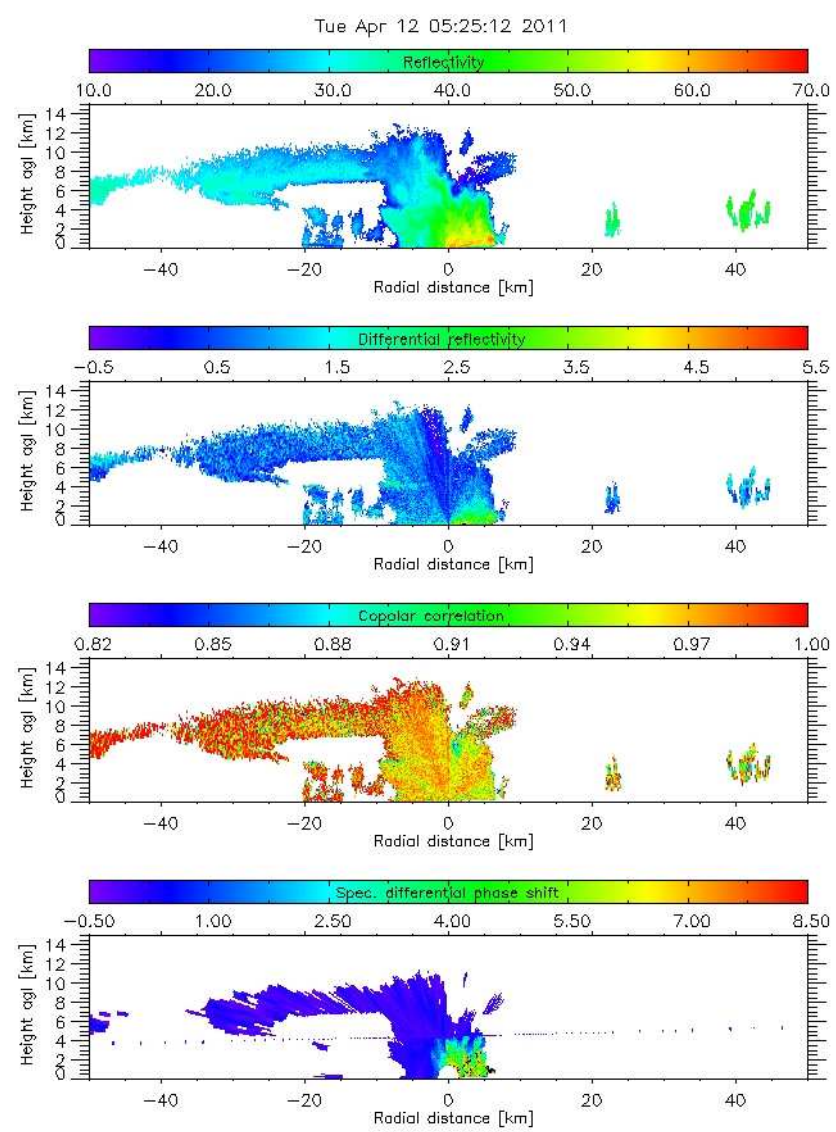

Fig. 2. Example of the polarimetric observables from one horizonto-horizon RHI sweep performed on 12 April 2011 in Fortaleza.

RHI pointed toward the sea in the direction where the main weather systems are expected to enter the continent. The volume was scanned with 13 elevations from $1.8^{\circ}$ to $21.4^{\circ}$ with a spacing of $1.1^{\circ}$ between the lowermost elevations and a spacing of $2.8^{\circ}$ between the uppermost elevations. The lowest elevation was set to $1.8^{\circ}$ since there was almost complete signal blocking below this angle. A fixed clutter filter width 


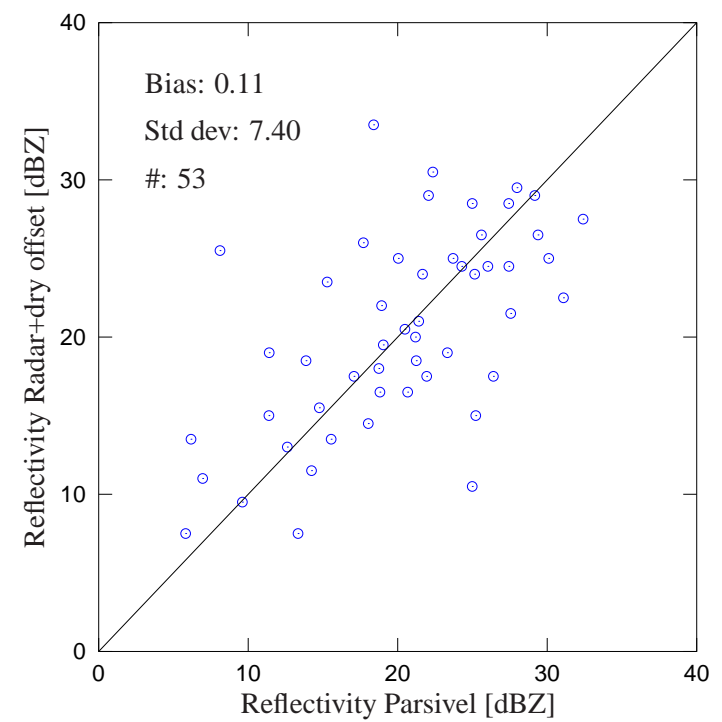

Fig. 3. Scatter plot of the offset corrected radar reflectivity vs. the reflectivity inferred from a collocated disdrometer. The data for the comparison was restricted to conditions where the radar itself was outside of the rain cell and the radome was dry. In addition, rain attenuation effects only played a minor role in the depicted data.

of $1.44 \mathrm{~m} \mathrm{~s}^{-1}$ was used and in addition, the strong signal blocking at low elevations acted like a clutter fence, hence the clutter contamination at higher elevations was low. One complete scanning cycle consisting of the mentioned two RHI scans, the volume scan and the $Z_{\mathrm{dr}}$ calibration was completed within 6 min.

The radar was calibrated by comparing disdrometer inferred reflectivities (see Sects. 2.3 and 3 for the details) with radar reflectivities measured at an elevation angle of $2.5^{\circ} \mathrm{ex}-$ tracted from an RHI scan at the distance of the disdrometer's location. Comparisons were restricted to events where it was certain that the radar was outside of the rain cell and that the radome of the radar was dry. In addition, the differential phase shift $\Psi_{\mathrm{dp}}$ had to be less than $10^{\circ}$ in order to limit rain attenuation effects to maximally $2 \mathrm{~dB}$. From this comparison, a calibration offset of $6.5 \mathrm{~dB}$ was determined. A scatter plot of the offset corrected radar reflectivities vs. the disdrometer inferred reflectivities that were selected under these conditions is depicted in Fig. 10. In the upcoming analysis, this offset is consequently added to the radar reflectivities, hence any other reflectivity offset will be due to additional effects that influence the calibration of the radar.

\subsection{Disdrometers}

The Parsivel laser disdrometers are capable of measuring the DSD of precipitation, i.e. the particle density per size class. With a horizontal laser beam it provides a sampling area of $54 \mathrm{~cm}^{2}$. When a particle crosses the laser beam, the intensity loss of the received laser signal as well as the time the particle remains in the beam are used to estimate the drop's equivolumetric diameter $D$ and its fall velocity $v$. The estimated sizes and fall speeds are stored in $32 \times 32$ matrix corresponding to 32 non-equidistant classes of diameter (from 0 to $25 \mathrm{~mm}$ ) and 32 non-equidistant classes of velocity (from 0 to $22.4 \mathrm{~m} \mathrm{~s}^{-1}$ ). From this raw spectrum diameter-velocity spectrum, not only the rain rate $R$ can be calculated but also the DSD $N(D)$, i.e. the drop concentration per volume and per diameter size class. Details on this procedure can be found in Battaglia et al. (2010) and Jaffrain and Berne (2011).

\section{T-matrix modeling}

The polarimetric variables $K_{\mathrm{dp}}\left[{ }^{\circ} \mathrm{km}^{-1}\right], Z_{\mathrm{h}, \mathrm{v}}\left[\mathrm{mm}^{6} \mathrm{~m}^{-3}\right]$, the differential phase shift on backscatter $\delta_{\mathrm{hv}}\left[{ }^{\circ}\right]$ as well as the specific one-way attenuation $A_{\mathrm{h}, \mathrm{v}}\left[\mathrm{dB} \mathrm{km}^{-1}\right]$ were calculated from the DSDs $(N(D))$ provided by the Parsivel disdrometer with following formulae (e.g. Bringi and Chandrasekar, 2001):

$$
\begin{aligned}
K_{\mathrm{dp}} & =\frac{180 \lambda}{\pi} \int_{D_{\min }}^{D_{\max }} \operatorname{Re}\left[f_{\mathrm{hh}}(D)-f_{\mathrm{vv}}(D)\right] N(D) \mathrm{d} D \\
Z_{\mathrm{h}, \mathrm{v}} & =\frac{4 \lambda^{4}}{\pi^{4}}\left|\frac{N_{\mathrm{r}}^{2}+2}{N_{\mathrm{r}}^{2}-1}\right| \int_{D_{\min }}^{D_{\max }}\left|s_{\mathrm{hh}, \mathrm{vv}}(D)\right|^{2} N(D) \mathrm{d} D \\
\delta_{\mathrm{hv}} & =\frac{180}{\pi} \arg \left[\int_{D_{\min }}^{D_{\max }} s_{\mathrm{hh}} s_{\mathrm{vv}}^{*} N(D) \mathrm{d} D\right] \\
A_{\mathrm{h}, \mathrm{v}} & =8.68 \lambda \int_{D_{\min }}^{D_{\max }} \operatorname{Im}\left[f_{\mathrm{hh}, \mathrm{vv}}\right] N(D) \mathrm{d} D,
\end{aligned}
$$

where $\lambda$ is the wavelength [mm], $N_{\mathrm{r}}$ the refractive index of water (dimensionless), $f_{\mathrm{hh}, \mathrm{vv}}$ the forward- and $s_{\mathrm{hh}, \mathrm{vv}}$ the backward-scattering amplitude in [m] at horizontal (hh) or vertical (vv) polarization. Re and Im denote the real and imaginary part, respectively, arg the argument and * the complex conjugate.

The scattering amplitudes can be calculated with the method known as T-matrix (Barber and Yeh, 1976) and the freely available Fortran implementation of Mishchenko and Travis (1998) was used for computation. The shape of the raindrops was approximated by an oblate spheroid. The axial ratio of the spheroid, being a function of the equivolumetric sphere diameter, were calculated with the models of Andsager et al. (1999), Brandes et al. (2002) and Thurai and Bringi (2005). Hence, for every individual drop one of the mentioned drop shape models was randomly chosen for the calculation of the vertical axis of the drop. This approach was chosen since it is not known which model performs best in the tropics. The angle of the main drop axis of 
the individual drops was modeled with a zero-mean Gaussian distribution with standard deviation of $10^{\circ}$ in order to take the canting angle effect into account (Park et al., 2005). The refractive index of water was calculated with the model of Meissner and Wentz (2004). Calculations were made at a frequency of $9.345 \mathrm{GHz}$, at a temperature of $300 \mathrm{~K}$ and with 23 diameter size classes ranging from $D_{\min }=0.062 \mathrm{~mm}$ to $D_{\max }=7.5 \mathrm{~mm}$. The size classes were predefined by the Parsivel instrument. The two smallest size classes $(0.062 \mathrm{~mm}$ and $0.187 \mathrm{~mm}$ ) are always empty due to signal-to-noise limitations for these small diameters, hence the lowermost detectable diameter is $0.312 \mathrm{~mm}$.

From the variables defined in Eqs. (1) and (3), $\Phi_{\mathrm{dp}}$ and $\Psi_{\mathrm{dp}}$ can be estimated as follows:

$$
\begin{aligned}
& \Phi_{\mathrm{dp}}=2 \int_{0}^{r_{\max }} K_{\mathrm{dp}}(r) \mathrm{d} r \\
& \Psi_{\mathrm{dp}}=\Phi_{\mathrm{dp}}+\delta_{\mathrm{hv}} .
\end{aligned}
$$

Relations between the rain rate $R$ and $Z_{\mathrm{h}}$ or $K_{\mathrm{dp}}$ are of particular interest for any application focusing on quantitative precipitation estimation (QPE). Since the body of literature on such relations for $\mathrm{X}$-band frequencies in tropical regions is small, we have plotted our modeling results in Fig. 4. The corresponding power law relations that were found by linearly fitting the logarithm of $R$ with the logarithm of the modeled $K_{\mathrm{dp}}$ and $Z_{\mathrm{h}}$ values adopt the following form:

$$
\begin{aligned}
R & =15.813 K_{\mathrm{dp}}^{0.774} \\
Z_{\mathrm{h}} & =214.18 R^{1.636} .
\end{aligned}
$$

It must be mentioned that the fitting procedure has a significant influence on the resulting parameters: As seen in Fig. 4, the density of the point cloud for low rain rate values is much higher than for high rain rates, which leads to large biases of the fit in the region of high rain rates. In order to overcome this effect, the rain rate was separated into different equally spaced $Z_{\mathrm{h}}$ or $K_{\mathrm{dp}}$ bins, respectively. Finally, in order to obtain Eqs. (7) and (8), the center of these rain rate bins, $\bar{R}$, and the average of $Z_{\mathrm{h}}\left(K_{\mathrm{dp}}\right)$ values within these bins, $\bar{Z}_{\mathrm{h}}\left(\bar{K}_{\mathrm{dp}}\right)$, were used for the fitting procedure instead of the original values. Nevertheless, relatively large errors in the estimation of $R$ are introduced if the relations (Eqs. 7 and 8) are used: We have found a standard deviation of $\sigma_{\mathrm{R}}^{Z_{\mathrm{h}}}=12.54 \mathrm{~mm} \mathrm{~h}^{-1}$ and a bias (retrieved minus true rain rate) of $\Delta_{\mathrm{R}}^{Z_{\mathrm{h}}}=0.800 \mathrm{~mm} \mathrm{~h}^{-1}$ if relation (Eq. 8) is used. The corresponding values for relation (Eq. 7) are $\sigma_{\mathrm{R}}^{K_{\mathrm{dp}}}=7.78 \mathrm{~mm} \mathrm{~h}^{-1}$ and $\Delta_{\mathrm{R}}^{K_{\mathrm{dp}}}=-0.0714 \mathrm{~mm} \mathrm{~h}^{-1}$.
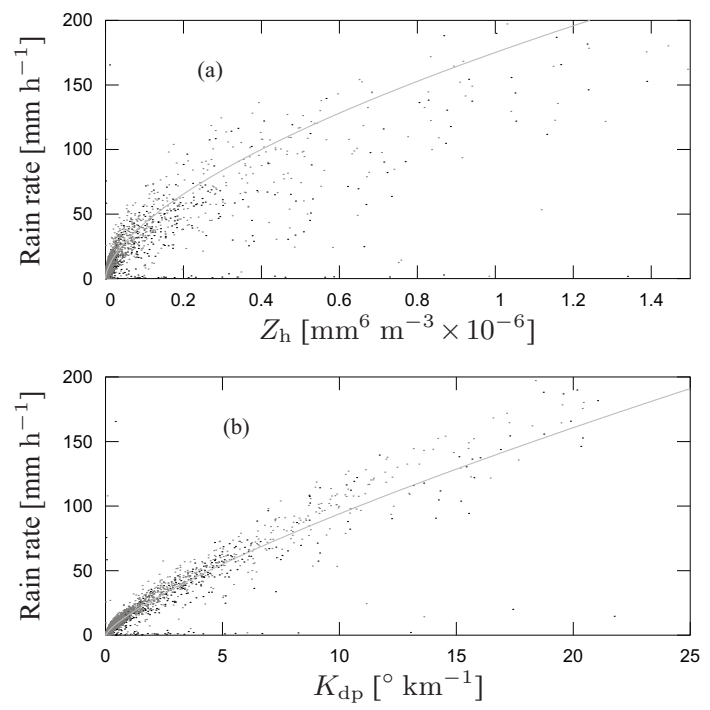

Fig. 4. (a) Scatterplot of the rain rate $R$ versus the radar reflectivity $Z_{\mathrm{h}}$ based on disdrometer derived DSD measurements. (b) Same as in panel (a) but for the rain rate $R$ versus $K_{\mathrm{dp}}$. The values of the shown power law fits are given in Eqs. (8) and (7).

\section{The extended Kalman filter algorithm}

In Schneebeli and Berne (2012), a method for the processing of polarimetric X-band radar data based on an extended Kalman filter was presented. With this method it is possible to correct X-band radar measurements for attenuation and differential attenuation caused by the rain medium but also to estimate the reflectivity offset that might be induced by a wet radome or by a miscalibration of the radar. In addition, the total differential phase shift on propagation measured by the radar, $\Phi_{\mathrm{dp}}$, can be separated into the differential phase shift on backscatter, $\delta_{\mathrm{hv}}$, while the sum of the two equals $\Psi_{\mathrm{dp}}$ without the measurement noise.

The extended Kalman filter (EKF) method employs relations between different polarimetric moments, relations of these moments between one range gate and the upcoming one, as well as covariances associated with these relations in order to constrain the solution space of the attenuation corrected reflectivities and the de-noised differential phase measurements. The basic principle behind the EKF method is that the different polarimetric moments can be estimated from each other in various ways and with varying accuracy. The knowledge on the accuracy of the estimator thereby determines how much it can be trusted. This principle gets enlightened if the estimation of $K_{\mathrm{dp}}$ is considered as an example: $K_{\mathrm{dp}}$ is internally estimated with (1) differentiating $\Phi_{\mathrm{dp}}$ and (2) with a direct relation between $K_{\mathrm{dp}}$ and $Z_{\mathrm{h}}$. Both ways of estimating $K_{\mathrm{dp}}$ have their associated uncertainties, which are used in the EKF method to weight the results of the two estimates. Relations between polarimetric moments are established with DSD measurement from Parsivel disdrometers 
Table 2. Parameters for the EKF method caluclated from the DSDs of three Parsivel disdrometers for a temperature of $300 \mathrm{~K}$.

\begin{tabular}{cccccccc}
\hline$\mu_{\mathrm{h}}$ & $\mu_{\mathrm{V}}$ & $\kappa_{\mathrm{h}}$ & $\kappa_{\mathrm{V}}$ & $\lambda_{\mathrm{h}}$ & $\lambda_{\mathrm{V}}$ & $\zeta$ & $\eta$ \\
\hline 0.212 & 0.169 & 44.03 & 42.35 & 1.283 & 1.176 & 0.670 & 1.612 \\
\hline
\end{tabular}

and subsequent T-matrix modeling of polarimetric parameters. For the EKF method to work, the parameters $\mu_{\mathrm{h}, \mathrm{v}}, \kappa_{\mathrm{h}, \mathrm{v}}$, $\lambda_{\mathrm{h}, \mathrm{v}}, \zeta$ and $\eta$ for the following relations must be found (for details the reader is referred to Schneebeli and Berne, 2012):

$$
\begin{aligned}
\Psi_{\mathrm{dp}}(i) & =-2 \Delta r 10^{\tilde{K}_{\mathrm{dp}}(i) / 10}+\Phi_{\mathrm{dp}}^{\prime}(i)+\delta_{\mathrm{hv}}(i) \\
\Psi_{\mathrm{dp}}(i+1) & =2 \Delta r 10^{\tilde{K}_{\mathrm{dp}}(i) / 10}+\Phi_{\mathrm{dp}}(i)+\delta_{\mathrm{hv}}(i) \\
\tilde{Z}_{\mathrm{h}}^{\mathrm{m}}(i) & =-\mu_{\mathrm{h}} \Phi_{\mathrm{dp}}(i)+\tilde{Z}_{\mathrm{h}}(i) \\
\tilde{Z}_{\mathrm{v}}^{\mathrm{m}}(i) & =-\mu_{\mathrm{v}} \Phi_{\mathrm{dp}}(i)+\tilde{Z}_{\mathrm{v}}(i) \\
-\kappa_{\mathrm{h}} & =\lambda_{\mathrm{h}} \tilde{K}_{\mathrm{dp}}(i)-\tilde{Z}_{\mathrm{h}}(i) \\
-\kappa_{\mathrm{v}} & =\lambda_{\mathrm{v}} \tilde{K}_{\mathrm{dp}}(i)-\tilde{Z}_{\mathrm{v}}(i) \\
0 & =\zeta\left(\tilde{Z}_{\mathrm{h}}(i)-\tilde{Z}_{\mathrm{v}}(i)\right)^{\eta}-\delta_{\mathrm{hv}}(i) \\
0 & =\Phi_{\mathrm{dp}}^{\prime}(i)-\Phi_{\mathrm{dp}}(i)-2 \Delta r 10^{\tilde{K}_{\mathrm{dp}}(i) / 10} .
\end{aligned}
$$

In the above equations, the range resolution is written as $\Delta r$; $Z_{\mathrm{h}}^{\mathrm{m}}$ and $Z_{\mathrm{v}}^{\mathrm{m}}$ are the measured reflectivities at $\mathrm{h}$ and $\mathrm{v}$ polarization, respectively. Quantities that are expressed in $\mathrm{dB}$ are denoted with a " $\sim$ " and $i$ is the range gate number. The notation $\Phi_{\mathrm{dp}}^{\prime}(i)=\Phi_{\mathrm{dp}}(i+1)$ was introduced in order to overcome ambiguities and in addition, a limited variation of $\delta_{\mathrm{hv}}$ from one gate to the next is considered, such that the relation $\delta_{h v}(i+1) \approx \delta_{\mathrm{hv}}(i)$ can be assumed. This approximation was found to be advantageous in the formulation of the retrieval scheme, since it allowed to save one additional state variable, which increased the overall accuracy of the method.

The parameters for the above equations are given in Table 2. They were deduced from the modeling results that are shown in Fig. 5. A similar procedure for the fitting of the polarimetric observables as described in the foregoing section was employed in order to increase the weighting of the (usually few) high values in the fitting process. For temperatures different from $300 \mathrm{~K}$, the EKF paramters can be calculated as a function of the temperature, as detailed in Appendix B.

The so-derived parameters are optimal for the one-month period in which disdrometer data were collected, where the term "optimal" is used with respect to the representativeness of the parameters. In the upcoming analysis, radar data that were acquired during the same period are treated with the EKF method that is based on these fitted parameters. This has some implications on the interpretation of the results, since such an optimal ensemble of parameter values might not always be available and hence values (either from the literature or inferred from disdrometer measurements that were performed at a different time and/or location) need to be used
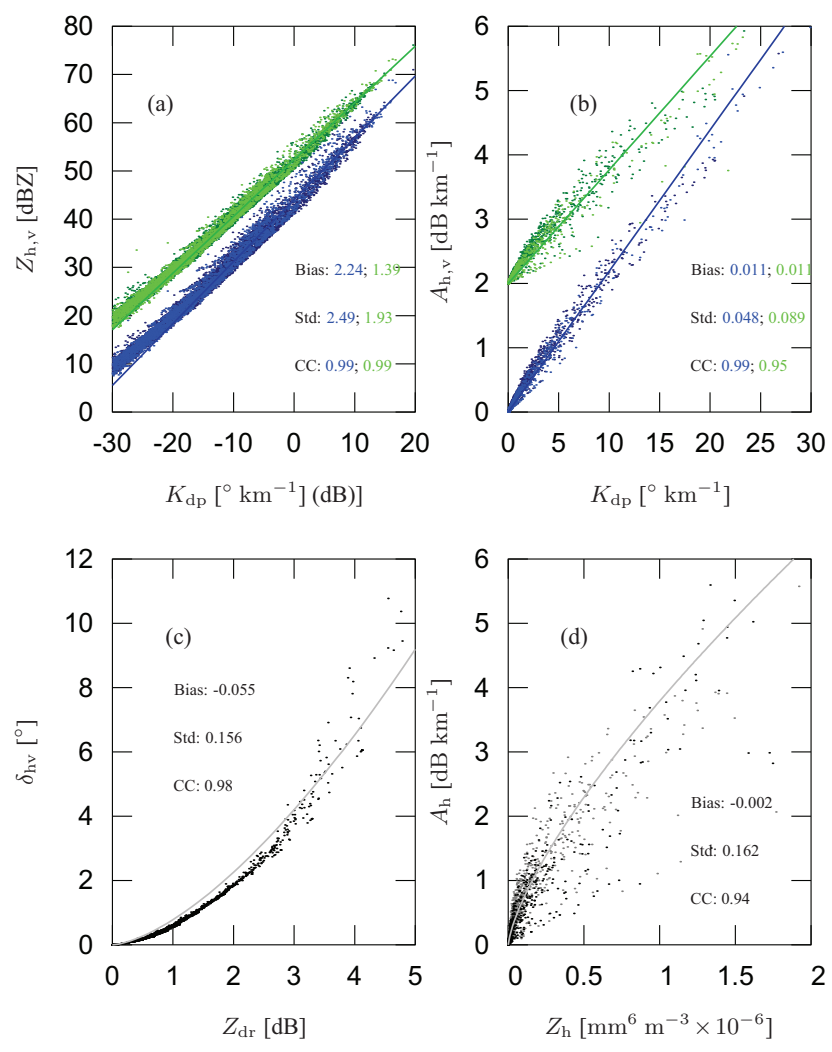

Fig. 5. T-matrix modeled relations between polarimetric variables: (a) Horizontally $\left(Z_{\mathrm{h}}\right.$; blue) and vertically $\left(Z_{\mathrm{V}}\right.$; green) polarized reflectivity versus $K_{\mathrm{dp}}$. $Z_{\mathrm{V}}$ is plotted with an artificial offset of $10 \mathrm{~dB}$ for clarity. (b) Horizontally $\left(A_{\mathrm{h}}\right.$; blue) and vertically $\left(A_{\mathrm{V}}\right.$; green) polarized specific attenuation versus $K_{\mathrm{dp}} . A_{\mathrm{V}}$ is plotted with an artificial offset of $2 \mathrm{~dB}$ for clarity. (c) Backscatter differential phase shift $\delta_{\mathrm{hv}}$ versus the differential reflectivity $Z_{\mathrm{dr}}$. d) $A_{h}$ versus $Z_{\mathrm{h}}$. Indicated in the plots are also the bias (i.e. the true minus the modeled value), with its standard deviation (Std) and correlation coefficient (CC). The sample size in all four plots was $N=19261$.

that have a reduced representativeness, which will negatively influence the performance of the applied algorithms.

The EKF method not only employs the relations given in Eqs. (9-16) as well as the covariance associated with these relations, it also makes use of the knowledge of the spatial variability of the polarimetric moments. Since it was found that the results of the EKF algorithm are not very sensitive to possible errors in the spatial representation to the polarimetric moments, spatial DSD data from Switzerland were used in order to calculate the gate-to-gate variability of the polarimetric observables. This data was obtained from a DSD simulator that is able to produce realistic spatial DSD fields (Schleiss et al., 2009) and hence delivers information on the spatial behavior of the polarimetric moments within a rain cell. This simulator was however constrained with spatial DSD measurements of Switzerland that are not necessarily representative for the locations in Brazil and it can also not 
be easily adapted to the different spatial DSD characteristics at the two locations.

One of the advantages of the EKF scheme lies in the fact that it can also be used to correct reflectivity offsets that are caused, for example, by a wet radome. Since the EKF scheme intrinsically calculates $K_{\mathrm{dp}}$ and $\Phi_{\mathrm{dp}}$, the two variables resulting from EKF calculations with different reflectivity offsets can be compared along one ray path and the comparison that exhibits the smallest absolute bias determines the reflectivity offset. This approach is somehow similar to the methods adopted in the so-called "self-consistency" attenuation correction algorithms (Bringi et al., 2001; Gorgucci et al., 2006). In Fig. 6, an example is given on how this algorithm works: in panel a, three pairs of $\int 2 K_{\mathrm{dp}} \mathrm{d} x$ and $\Phi_{\mathrm{dp}}$ profiles that were inferred with the EKF algorithm are shown. The only difference between the pairs of profiles is the initial reflectivity offset (indicated at the beginning of the range profiles) that was added to the measured reflectivity prior to the treatment with the EKF algorithm. It is seen that a reflectivity offset of $10 \mathrm{~dB}$ leads to the smallest bias between the curve pairs compared to the two other offsets ( 5 and $15 \mathrm{~dB}$ ). In panel $b$, the absolute bias between the curve pairs is plotted against the initial reflectivity offset. The minimum of the curve shown in this plot (around $10 \mathrm{~dB}$ ) determines the final radar reflectivity offset.

In Fig. 7, an example for the performance of the EKF and the ZPHI algorithm is given. In this plot, the original (offset corrected) reflectivity and differential reflectivity together with the attenuation corrected reflectivities as a function of the radar range are depicted. In addition, $K_{\mathrm{dp}}$ inferred from the EKF scheme as well as the original measured differential phase together with the EKF calculated $\Phi_{\mathrm{dp}}$ and $\delta_{\mathrm{hv}}$ are shown. It is seen that the two attenuation correction schemes exhibit a considerable bias at the end of the range profile and it is not yet clear what is causing this difference. However, in panel $\mathrm{d}$ it is seen that $\delta_{\mathrm{hv}}$ and $\Phi_{\mathrm{dp}}$ add up nicely to the measured phase $\Psi_{\mathrm{dp}}$, which is a good indication that the algorithm performs well.

\section{Attenuation correction with ZPHI}

In order to compensate the energy loss due to extinction and attenuation the radar beam suffers when it propagates through the rain medium, the well known ZPHI algorithm (Testud et al., 2000) was implemented. With this method, attenuation and differential attenuation is calculated as

$$
\begin{aligned}
A_{\mathrm{h}}(i) & =\frac{Z_{\mathrm{h}}(i)^{b}}{I\left(0, i_{1}\right)+\left(10^{0.1 b \gamma \Delta \Phi_{\mathrm{dp}}}-1\right) I\left(i, i_{1}\right)} \\
& \times\left(10^{\left.0.1 b \gamma \Delta \Phi_{\mathrm{dp}}-1\right)}\right. \\
A_{\mathrm{dp}} & =\alpha A_{\mathrm{h}}^{\beta}
\end{aligned}
$$

with
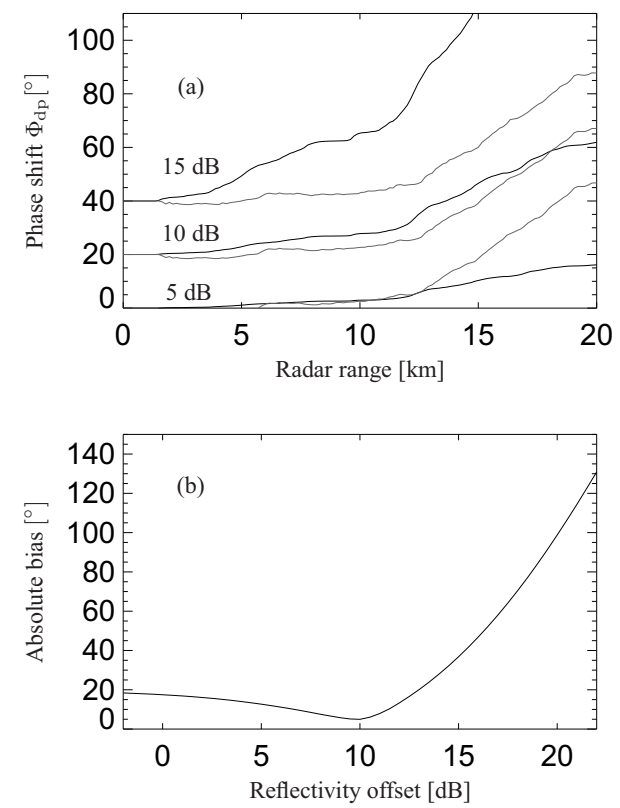

Fig. 6. (a) Range integrated $K_{\mathrm{dp}}$ (black lines) and $\Phi_{\mathrm{dp}}$ (grey lines) both inferred from the EKF algorithm and calculated with three different reflectivity offsets. The three curves associated with the three reflectivity offsets are plotted with a phase offset of $20^{\circ}$. (b) The abolute bias between the black and the grey lines from the upper panel as a function of the reflectivity offset.

$I\left(i, i_{1}\right)=0.46 b \sum_{j=i}^{i_{1}} Z_{\mathrm{h}}(j)^{b} \Delta r$.

Hereby, $i_{1}$ is the last gate of the range profile, $\Delta \Phi_{\mathrm{dp}}$ is the differential phase from 0 to $i_{1}, b$ is defined as the exponent of the relation

$A_{\mathrm{h}}(i)=x Z_{\mathrm{h}}(i)^{b}$

and $\gamma$ stems from the relation

$A_{\mathrm{h}}(i)=\gamma K_{\mathrm{dp}}(i)$.

For simplification reasons and in contrast to the original ZPHI method, it is assumed that $\alpha$ in Eq. (18) is constant over the whole range profile, i.e. that only one homogeneous rain cell is present along the range profile.

The performance of ZPHI solely depends on the accuracy of the estimation of the $b, \beta, \alpha$ and $\gamma$ parameters. These parameters were calculated as described in the foregoing section by applying the T-matrix method to DSDs measured with a disdrometer. The calculations corresponding to the relations of Eqs. (20) and (21) are shown in Fig. 5d and 5b, respectively. Also note that the parameter $\gamma$ given in Eq. (21) is similar to the parameter $\mu$ in Eq. (11).

The numerical values of the determined parameters are given in Table 3. 

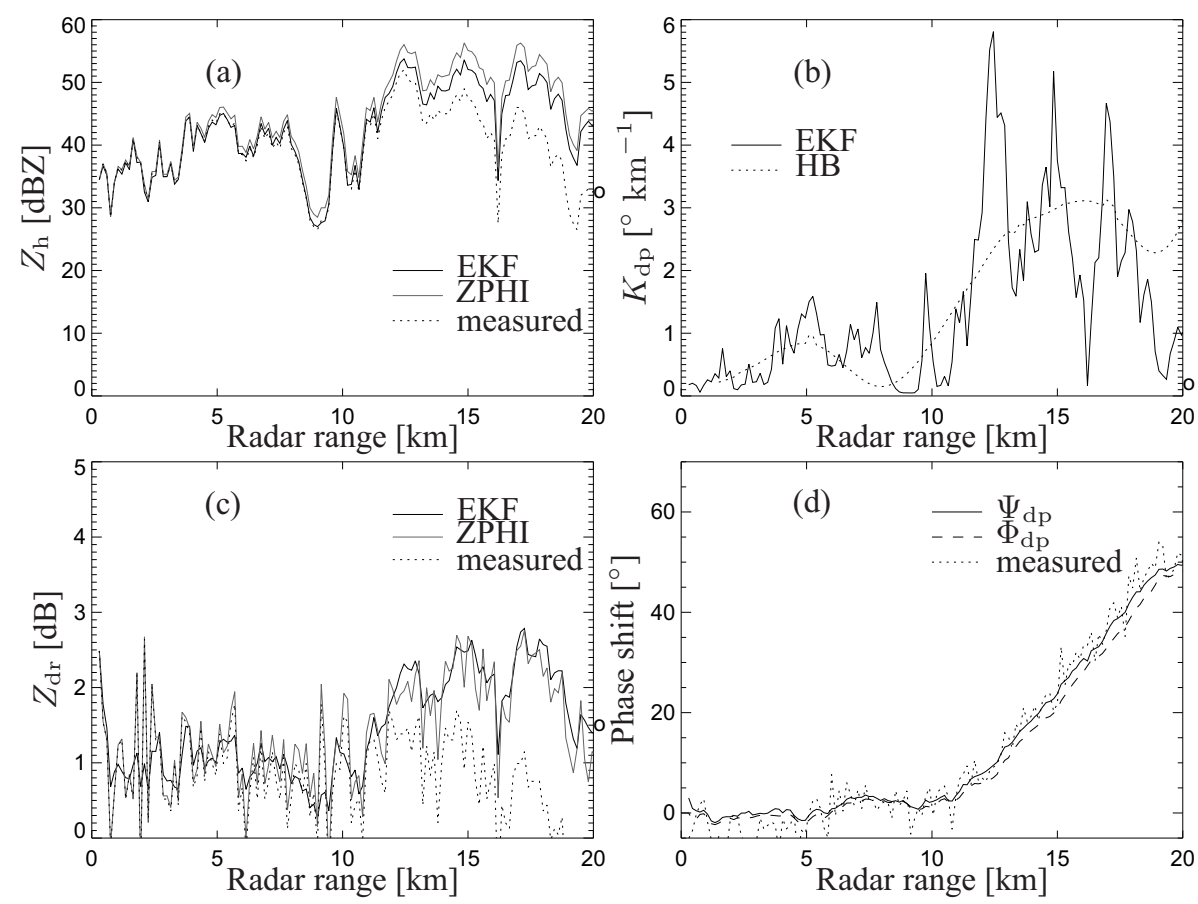

Fig. 7. (a) Profile of the measured (dotted) and attenuation corrected (ZPHI: grey line, EKF: black line) $Z_{\mathrm{h}}$. (b) Ray profile of $K_{\mathrm{dp}}$ estimated with EKF and with the standard smoothing algorithm (HB; Hubbert and Bringi, 1995). (c) Same as in (a) but for $Z_{\mathrm{dr}}$. (d) Ray profiles of the measured (dotted) and retrieved (black line) total differential phase $\Psi_{\mathrm{dp}}$, which is the sum of the retrieved $\delta_{\mathrm{hv}}$ and $\Phi_{\mathrm{dp}}$ (dashed). The circle "o" at the end of the profiles in panels (a), (b) and (c) indicates the corresponding disdrometer value.

Table 3. Parameters for the the ZPHI attenuation correction method caluclated from the DSDs of three Parsivel disdrometers.

\begin{tabular}{cccc}
\hline$\gamma$ & $b$ & $\alpha$ & $\beta$ \\
\hline 0.212 & 0.724 & 0.117 & 1.265 \\
\hline
\end{tabular}

\section{Differential reflectivity bias correction}

In addition to the reflectivity offset, there exists also a bias in $Z_{\mathrm{dr}}$ that needs to be removed before the data can be further processed. Traditionally, this offset is determined by pointing the antenna in vertical direction and rotating it around the azimuthal axis (Seliga et al., 1979; Gorgucci et al., 1999). Since the rain drops at this incidence angle should not exhibit any differential polarization, the $Z_{\mathrm{dr}}$ data that is acquired in this manner can be averaged (over the range gates and the azimuthal angles), which should lead to a value that corresponds to the difference between the $\mathrm{H}$ - and the $\mathrm{V}$ channel. The $Z_{\mathrm{dr}}$ offset shows a distinct dependence on the azimuth angle as seen in Fig. 8a. As stated in Gorgucci et al. (1999), this azimuthal dependence is usually due to clutter contamination from side lobes, but it should not hamper the applicability of the accuracy of the $Z_{\mathrm{dr}}$ calibration procedure if the data is averaged over one full azimuthal cycle. With this method applied to data that consisted of 110 full azimuthal cycles, the mean $Z_{\mathrm{dr}}$ offset was determined to $Z_{\mathrm{dr}}^{\text {off }}=-0.34 \mathrm{~dB}$.

In Fig. 8b it is seen that the $Z_{\mathrm{dr}}$ offset is relatively stable in time (with just some outliers that are probably due to atmospheric effects like partial beam filling at the end or the beginning of a rain event). It is therefore suggested to determine the $Z_{\mathrm{dr}}$ offset just once or only from time to time and then assume the offset to be stable over time. At the moment we believe that with this procedure more accurate $Z_{\mathrm{dr}}$ values can be obtained than with a regular offset correction.

Because of the issue with the damaged radome, it is thought to be advantageous to additionally calibrate $Z_{\mathrm{dr}}$ with a method that does not rely on data gathered at vertical incidence, since this damaged radome might negatively influence the aforementioned $Z_{\mathrm{dr}}$ calibration technique that uses the vertically pointing and rotating antenna. If the antenna points at a horizontal direction into a rain field, $Z_{\mathrm{dr}}$ values are usually unknown a priori and can therefore not be used to calibrate the radar. There is however one exception: if the reflectivity values are low and the data is not affected by attenuation, then the $Z_{\mathrm{dr}}$ values have to be very close to zero. This assumption was tested with T-matrix simulations and the mean and standard deviation of $Z_{\mathrm{dr}}$ in such low reflectivity conditions was calculated to a value of $Z_{\mathrm{dr}}=0.1 \pm 0.052 \mathrm{~dB}$. If it is now possible to find such values (low $Z_{\mathrm{h}}$, no attenuation), the corresponding $Z_{\mathrm{dr}}$ values can be set to $Z_{\mathrm{dr}}^{\text {low }}=0.10$, which determines the $Z_{\mathrm{dr}}$ offset. To do so, it was searched for 

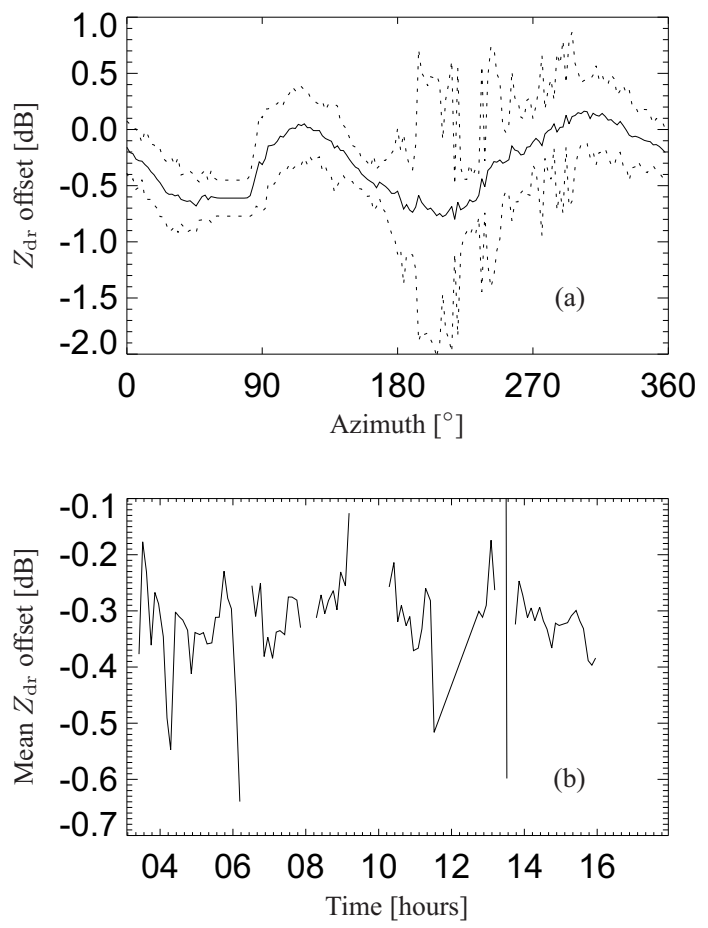

Fig. 8. (a) $Z_{\mathrm{dr}}$ offset as a function of the azimuth angle measured over a period of $12 \mathrm{~h}$ on 12 April 2011. The black line shows the mean offset over this period and the dotted lines indicate the standard deviation. (b) Time series of the mean $Z_{\mathrm{dr}}$ offset that was calculated from the average over all azimuth directions.

these conditions by applying certain criteria: first, the $Z_{\mathrm{h}}$ values had to be low and fulfill the condition $Z_{\mathrm{h}} \leq 10 \mathrm{dBZ}$. Second, the signal-to-noise ratio (SNR; it was internally calculated by the Gematronik radar software) had to be above $3 \mathrm{~dB}$ in order to reduce noise effects on $Z_{\mathrm{dr}}$. Finally, only values in regions where $\Psi_{\mathrm{dp}}$ along a ray profile did not exceed $10^{\circ}$ were considered. This threshold ensured that $Z_{\mathrm{dr}}$ values only suffered from maximally $0.2 \mathrm{~dB}$ attenuation. It was assumed that $Z_{\mathrm{dr}}$ values measured under these conditions suffered from $Z_{\mathrm{dr}}^{\text {att }}=0.1 \mathrm{~dB}$ on average, i.e. half of the maximum attenuation value. In Fig. 9 a histogram of $Z_{\mathrm{dr}}$ values is shown that were measured under the mentioned conditions. Data used for this figure were collected from 12-22 April 2011. With the mean value of $Z_{\mathrm{dr}}^{\text {mean }}=0.031 \mathrm{~dB}$, we can determine the $Z_{\mathrm{dr}}$ offset to $Z_{\mathrm{dr}}^{\text {off }}=Z_{\mathrm{dr}}^{\text {mean }}+Z_{\mathrm{dr}}^{\text {att }}-Z_{\mathrm{dr}}^{\text {low }}=0.031 \mathrm{~dB}$. This result is rather surprising, since it indicates that $Z_{\mathrm{dr}}$ of the radar was already well calibrated and that the problem with the radome leads to a bias in the $Z_{\mathrm{dr}}$ calibration of approximately $-0.3 \mathrm{~dB}$. Due to these results, the original $Z_{\mathrm{dr}}$ calibration was considered to be accurate and also stable in time and was therefore assumed to be constant throughout the campaign.

Although other methods exist in the literature to determine the $Z_{\mathrm{dr}}$ offset without pointing to zenith direction (Ryzhkov et al., 2005; Bechini et al., 2008), these methods require

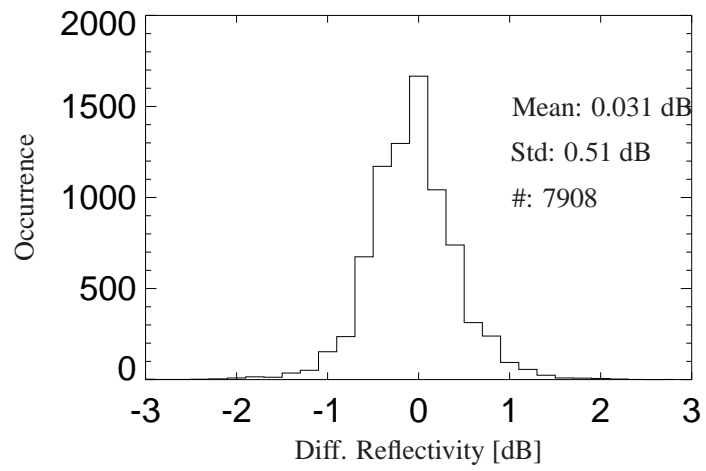

Fig. 9. Histogram of $Z_{\mathrm{dr}}$ values found in conditions of low reflectivity and low attenuation.

stratiform precipitation, while during our campaign mostly convective situations were encountered.

Due to the relatively large standard deviation of $\sigma_{Z_{\mathrm{dr}}}=0.51 \mathrm{~dB}$ that is found in the histogram, it is questionable if this method is accurate enough for calibrating $Z_{\mathrm{dr}}$. This high standard deviation is most likely induced by very low reflectivities that exhibit a low signal-to-noise ratio. It could however also be caused by polarization leakage effects (Wang and Chandrasekar, 2006) that can lead to considerable biases in $Z_{\mathrm{dr}}$ (up to $1 \mathrm{~dB}$ ). Especially simultaneously transmitting radars like the one that is employed in the study at hand are prone to such errors in $Z_{\mathrm{dr}}$.

\section{Results and discussion}

\subsection{Reflectivity offset due to radome attenuation}

In Sect. 4 it was shown how the reflectivity offset due to miscalibration and/or radome effects can be determined with the EKF method. However, a minimal total differential phase shift $\left(\Phi_{\mathrm{dp}}^{\max }\right)$ of $20^{\circ}$ along the radar ray path is necessary for the method to work properly, such that the calibration offset can be determined accurately. It is very common that $\Phi_{\mathrm{dp}}^{\max }$ is below the proposed threshold and then the radome offset has to be determined differently, which is detailed in the following. from Fig. 5 it is seen that there is a close relation between the reflectivity $\tilde{Z}_{\mathrm{h}, \mathrm{v}}$ and $\tilde{K}_{\mathrm{dp}}$. If $\Phi_{\mathrm{dp}}^{\max }$ along a ray path is low, i.e. if the reflecivities along this path only suffer from low attenuation, this relation can be used to determine the reflectivity offset by calculating $\tilde{Z}_{\mathrm{h}, \mathrm{v}}$ from $\tilde{K}_{\mathrm{dp}}$ employing the relations given in Eqs. (13) and (14) and then comparing the results to the measured reflectivities $\tilde{Z}_{\mathrm{h}, \mathrm{v}}^{\mathrm{m}}$. This method, which will be called " $K_{\mathrm{dp}}$ method" hereupon, is employed where $\Phi_{\mathrm{dp}}^{\max }<20^{\circ}$. Due to the large scatter that is inherent in the relations of Eqs. (13) and (14), the $K_{\mathrm{dp}}$ method is expected to provide less accurate estimates of the reflectivity offset than the EKF method. Attenuation effects that can negatively influence the $K_{\mathrm{dp}}$ method are overcome by applying ZPHI 
attenuation correction to the reflectivities prior to determining the reflectivity offset. Also note that the $K_{\mathrm{dp}}$ method depends on the elevation angle due to the elevation dependence of $K_{\mathrm{dp}}$. Since this method is applied only for elevation angles $\phi<6^{\circ}$, this dependence is negligible and hence no specific elevation angle correction was applied.

The $K_{\mathrm{dp}}$ method has certain similarities with the EKF method, since both methods are based on the self-consistency between different polarimetric observables. The EKF method however estimates $\Phi_{\mathrm{dp}}$ and $K_{\mathrm{dp}}$ individually as two different state variables, although they are linked to each other within the retrieval framework. Both $\Phi_{\mathrm{dp}}$ and $K_{\mathrm{dp}}$ estimated with the EKF depend on the reflectivity calibration and the consistency between the two variables is highest when the radar is perfectly calibrated (as seen in Fig. 6). In contrast, the $K_{\mathrm{dp}}$ method relies only on the relatively loose relation between $Z_{\mathrm{h}}$ and $K_{\mathrm{dp}}$ that is shown in Fig. 5, without any further constraints.

In order to get an example of the variability of the reflectivity offset, the radome attenuation has been determined in azimuth and elevation direction for 12 April 2011, 05:20 UTC, and the result is given in Fig. 10 where the reflectivity offset as a function of the azimuth angle is shown in panel a and as a function of the elevation angle in panel $b$. At the time of the observation, very intense rainfall was encountered right above the radar. In panel a, only the EKF method was employed to compile the plot in order to eliminate the influence of the choice of the method on the result. No offset was calculated for directions where a value of $\Phi_{\mathrm{dp}}^{\max }=20^{\circ}$ could not be reached, and in the graphical representation the values in these regions were simply interpolated linearly.

The plot in panel $b$ is differently compiled: since the EKF method was developed for horizontal incidence of the radar beam to the rain drops, the relations given in Eqs. (9)-(15) are not valid anymore for too high elevation angles. T-matrix simulations showed that the difference between an elevation angle of $45^{\circ}$ and $0^{\circ}$ leads to biases in the EKF parameters (given in Table 2) of up to $100 \%$ (for the $\mu_{\mathrm{h}, \mathrm{v}}$ parameters), which is not acceptable for the scope of the application. Hence, the EKF parameters had to be corrected for higher elevation angles with a procedure that is detailed in Appendix A. For elevation angles higher than $45^{\circ}$, the EKF method becomes inaccurate since the polarimetric signature of the rain vanishes with increasing elevation angle (although the parameters were elevation angle corrected). Therefore, the EKF method was employed for the calculation of the reflectivity offset only up to an elevation angle of $45^{\circ}$. The values for higher angles were calculated with the assumption that the reflectivity at the closest meaningful range gate (being located at $300 \mathrm{~m}$ distance from the radar) linearly varies between the elevation angles of $45^{\circ}$ and $135^{\circ}$. Although the spatial extent of the volume that is defined with these conditions is low, the assumption of a linearly varying reflectivity right above the radar introduces a considerable error that is due to the high spatial variability of rainfall (Jaffrain
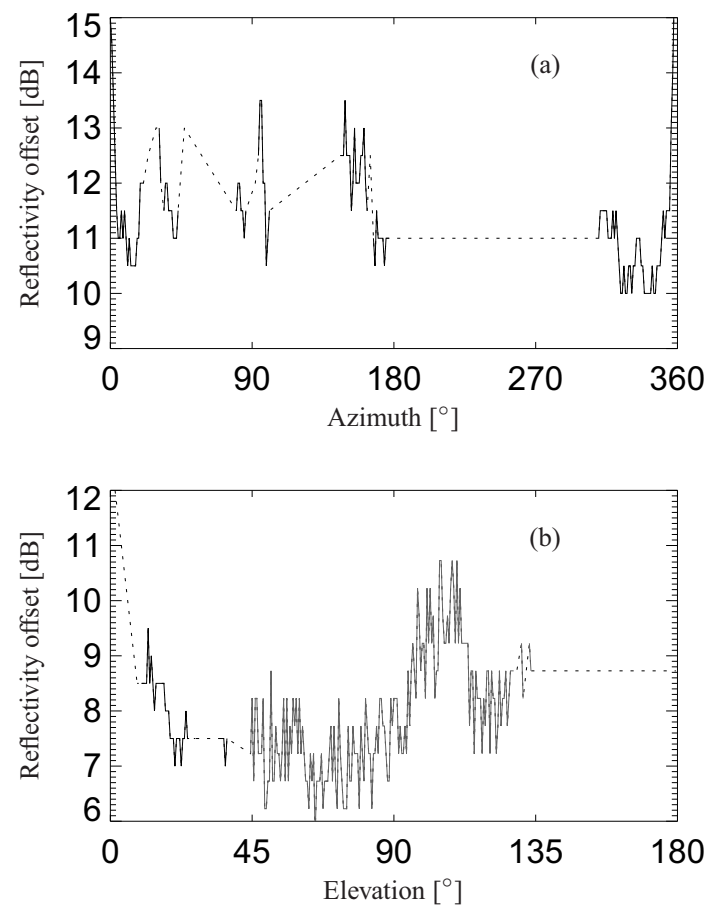

Fig. 10. (a) Reflectivity offset as a function of the azimuth angle, measured on 12 April 2011, 05:20 UTC. The black lines indicate values that are determined with the EKF algorithm, while dashed lines show interpolated values. (b) Same as in (a) but as a function of the elevation angle. In addition to the plot in panel (a), the grey line shows values that are determined from the scaling of the reflectivity value at the first range gate as described in Sect. 7.1.

et al., 2011). With the stochastically simulated rain fields of Schleiss et al. (2009), it was possible to estimate the standard error of $Z_{\mathrm{h}}$ in zenith direction determined with linear interpolation between elevation angles of $45^{\circ}$ and $135^{\circ}$ to $\sigma_{Z_{\mathrm{h}}}=2.67 \mathrm{~dB}$ in strongly convective situations. Since $\sigma_{Z_{\mathrm{h}}}$ rapidly decreases towards lower elevations angles, the described method is nevertheless found to be useful. The result of this procedure is shown in Fig. 10b, where the reflectivity offsets determined with the EKF method are plotted in black and those interpolated with the method described above are plotted in grey.

It is seen that around $110^{\circ}$ elevation, a local maximum of the radome attenuation is encountered. It is very likely that this maximum is caused by the damaged radome that was fixed with fiberglass and glue, since the damage is located in the direction where the attenuation maximum is encountered. Apparently, the performance of the fixed radome in terms of transparency is slightly worse than the undisturbed radome. However, the most striking feature in this graph are the high values around $11 \mathrm{~dB}$ that were measured for the reflectivity offset. These measurements emphasize that the radar data without radome correction cannot be used for any quantitative analysis if the radome is under influence of heavy rain. 
In azimuth direction, it is seen that the variation of the offset is relatively large, ranging from 10 to $13.5 \mathrm{~dB}$ (with one orientation that exhibits a value of $15.5 \mathrm{~dB}$ ). The variability in azimuth could be related to nonuniform wetting or nonuniform drying of the radome, which is probably caused by wind patterns. Due to this high variability, it is not recommended to apply a general correction that is valid for all the azimuthal directions. In order to get observations of high quality and maximum accuracy, every direction should be individually corrected for radome induced offsets.

In Bechini et al. (2010) a function is reported that links the radome attenuation of the same Gematronik X-band radar with the rain intensity that was measured at the radar location. This function for the radome attenuation $L_{\text {rad }}$ reads:

$L_{\mathrm{rad}}=2\left(-0.34+1.75 R^{1 / 3}\right)$,

with the rain rate $R$ [mm] and $L_{\mathrm{rad}}$ in [dB]. Unfortunately, we did not deploy an instrument to measure $R$ at the radar site, which makes it a bit more difficult to compare our findings with Eq. (22). In order to estimate $R$ at the radar site, the offset corrected reflectivity data from the first range gates when the antenna pointed at vertical direction were taken and averaged. Then, $R$ was estimated with the $Z-R$ relation given in Eq. (8) that was found from the T-matrix simulations shown in Fig. 5. The reflectivity offsets determined with the two different methods are shown as a scatter plot in Fig. 11. It is obvious that this procedure inherently introduces a certain correlation between the two different offset estimates, because the offset calculated with EKF is also indirectly used for the calculation of the offset with Eq. (22). This correlation was however found to be low (with a correlation coefficient of 0.25 ) when random values for the reflectivity $Z_{\mathrm{h}}$ and for the EKF offset were chosen.

A clear correlation between the two methods is observed, specifically at higher offset values. Towards lower offset values, the relation between the two methods is more scattered. The reason for this behavior is found in the fact that the radome can remain wet even when it is not raining right at the location of the radar. In this case, i.e. if the radome is still wet but the rain intensity is zero, the Bechini method also estimates the radome offset to zero. Another error source of the Bechini method is the imperfection of the $R$ estimation due to the scatter of the $Z-R$ relationship. However, if the rain rates were estimated directly at the radar site with a pluvioor disdrometer, this error could be mitigated. The Bechini method also only estimates the reflectivity offset due to the wet radome. Any calibration effects that are caused by a different effect (e.g. variations in the receiver sensitivity, variations in the transmitted power) cannot be tackled with this method and are therefore expected to contribute to the scatter found in Fig. 11a.

There is not only considerable variation of the radome offset in azimuth and elevation direction, but also in time, as it is seen in Fig. 11b, where a time series of the reflectivity offset
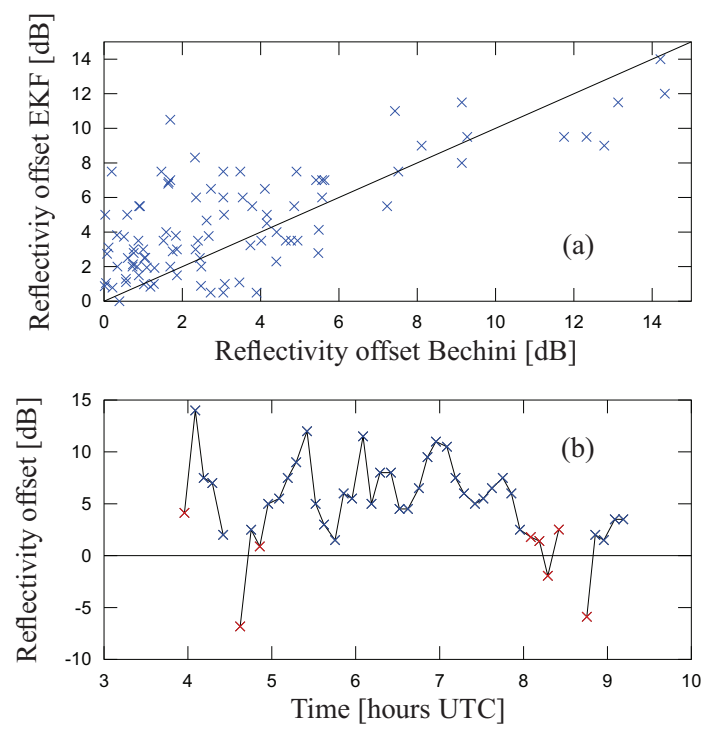

Fig. 11. (a) Scatter plot of the reflectivity offsets determined with the method of Bechini et al. (2010) and the EKF algorithm. (b) Time series of reflectivity offsets determined with the EKF method (blue crosses) and with the $K_{\mathrm{dp}}$ method (red crosses).

determined in one single direction is plotted. For this plot, the $K_{\mathrm{dp}}$ method was employed where the EKF method could not be applied. It is seen that the reflectivity offset values determined with the EKF method are generally higher, since high values of $\Phi_{\mathrm{dp}}^{\max } \leq 20^{\circ}$, being a prerequisite for the EKF method to work, are more likely to be found where also the rain intensity above the radar is high, i.e. where the radome is wet and therefore induces high signal attenuation. However, this is only a general rule, since it is possible to have a large $\Phi_{\mathrm{dp}}^{\max }$ in one direction even if the radar is located outside of a rain cell.

Given the large values found for the radome attenuation, it remains to be investigated if a non-uniformly wet radome causes differential attenuation as well. This was checked by separating the distribution of $Z_{\mathrm{dr}}$ values that are given in Fig. 9 into different radome attenuation classes in order to establish a possible relation between the radome attenuation and the radome differential attenuation. The values for the radome attenuation were thereby calculated with the EKF method. No matter how the classification into different radome attenuation bins was chosen, the $Z_{\mathrm{dr}}$ offset remained between 0.1 and $-0.15 \mathrm{~dB}$. It is therefore concluded that the water film on the radome only marginally influences the $Z_{\mathrm{dr}}$ calibration.

\subsection{Rain attenuation correction}

With the parameters given in Table 3 and the Eqs. (17) and (18), the attenuation $A_{\mathrm{h}}$ and differential $A_{\mathrm{dp}}$ can be calculated at every range gate along a ray path. Together with the radome offset correction and the $Z_{\mathrm{dr}}$ offset correction, the 

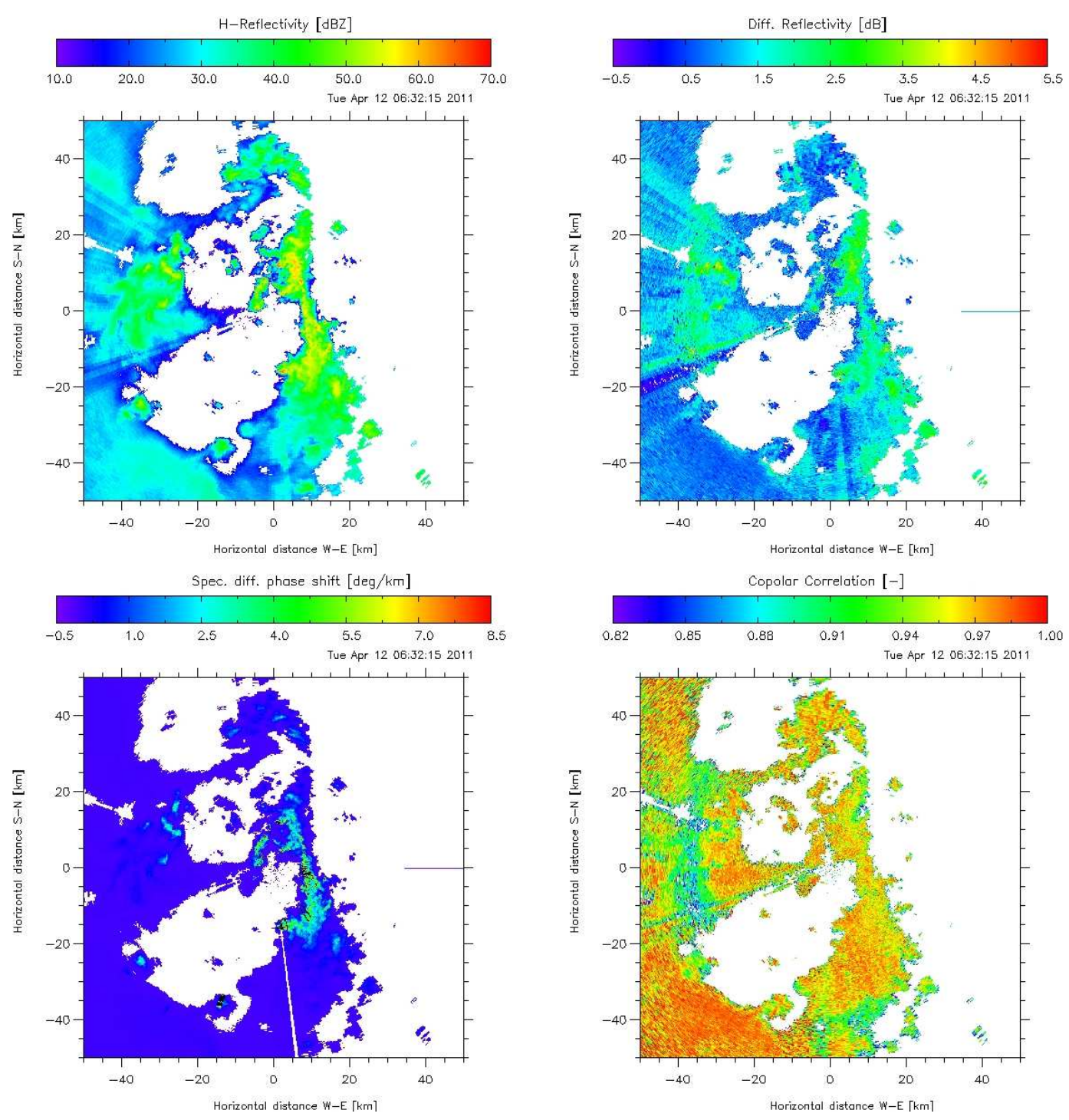

Fig. 12. Example of the polarimetric observables from one PPI scan performed on 12 April 2011 at an elevation of $5.3^{\circ}$. Note that the streak around $250^{\circ}$ is associated to an antenna of a radio telescope that was located at close distance to the radar.

data that are treated in this manner can be regarded as fully corrected and are ready for further scientific or operational use.

In Fig. 12, the polarimetric variables of one PPI scan that underwent the full data preprocessing chain is shown. Attenuation correction was performed with the ZPHI algorithm and $K_{\mathrm{dp}}$ stems from the EKF algorithm. The copolar correlation coefficient $\rho_{\mathrm{hv}}$ did not undergo any pre-processing and is taken as provided by the Gematronik radar.

In the shown reflectivity and differential reflectivity data, there remain still certain radial "streaks", especially in the directions west and south of the radar. These streaks indicate that the radome offset correction in these specific directions was determined with low accuracy, which causes inconsistencies in the $Z_{\mathrm{h}}$ and $Z_{\mathrm{dr}}$ field from one direction to the other.
Discrepancies that can cause these streaks are numerous: it is possible that the method for determining the reflectivity offset changes from one azimuthal direction to the neighboring one from the EKF method to the $K_{\mathrm{dp}}$ method or vice versa. Partial beam blockage caused by ground clutter is expected to hamper the accuracy of the EKF method, since this effect causes the relations given in Eqs. (9)-(16) to become invalid or at least erroneous, which has a negative effect on the accuracy of the determination of the reflectivity offset as a consequence. However, the general visual impression of the reflectivity fields suggest that the attenuation correction procedure leads to meaningful results.

A further and rather extreme example that illustrates the necessity of radome and rain attenuation is shown in the Fig. 13: in panel c of this figure, which shows the reflectivity 


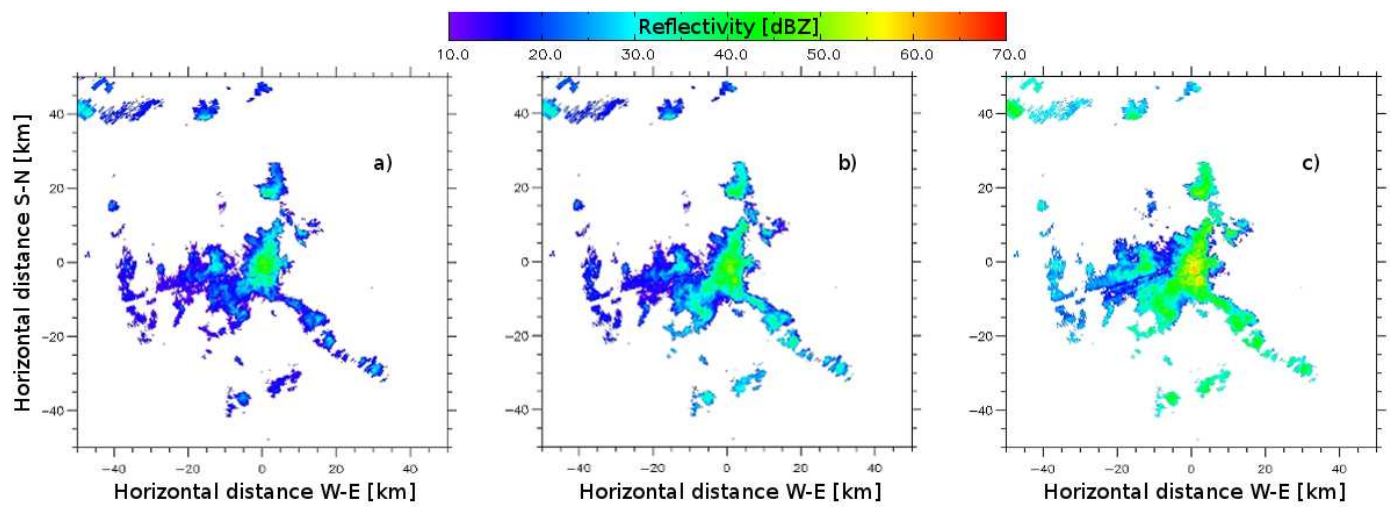

Fig. 13. (a) PPI scan showing uncorrected reflectivity. (b) The same scan that is shown in (a) but after rain attenuation correction with the ZPHI algorithm. (c) As in (b) but with additional radome attenuation correction.

after all the pre-processing steps, it can be seen that a very strong rain cell is located right at the radar location. This rain cell causes very strong radome attenuation and hence the uncorrected data, as shown in panel a, differs considerably from the corrected one.

\subsection{Comparisons with a disdrometer}

The algorithm's performance can be quantitativley analyzed by comparing the corrected radar polarimetric moments with such moments that are modeled from DSD measurements of a Parsivel disdrometer located at a distance of $20 \mathrm{~km}$ from the radar. To do so, from the RHI scan that was performed in the disdrometer's azimuthal direction $\left(316.7^{\circ}\right)$, the corresponding range gate was extracted for an elevation angle of $2.5^{\circ}$. This angle corresponded to the lowermost elevation that was completely unspoiled from ground clutter contamination.

The polarimetric moments $Z_{\mathrm{h}}, Z_{\mathrm{dr}}$ and $K_{\mathrm{dp}}$ inferred from the disdrometer DSD measurements were calculated according to the procedure detailed in Sect. 3 and compared to the attenuation corrected radar measurements. The reflectivity offset was calculated with the EKF method, where applicable, and with the $K_{\mathrm{dp}}$ method for the remaining cases. The EKF algorithm was also used to determine rain attenuation corrected $Z_{\mathrm{dr}}$ and $Z_{\mathrm{h}}$ as well as $K_{\mathrm{dp}}$ values. In addition, $Z_{\mathrm{h}}$ and $Z_{\mathrm{dr}}$ were also corrected for rain attenuation with the ZPHI method, and $K_{\mathrm{dp}}$ was determined with the traditional algorithm of Hubbert and Bringi (1995) in order to assess the performance of the different methods.

In Fig. 14, a scatter plot with the horizontal reflectivity inferred from Parsivel data versus the uncorrected and corrected radar measurements is shown, where the radar measurements were corrected with the EKF algorithm. Since two methods for the correction of the reflectivity offset were employed, the corrected radar measurements are represented with a "o" where the offset was deduced with the EKF method and with an " $\times$ " where the $K_{\mathrm{dp}}$ method was employed. Similarly to the findings in the previous section,

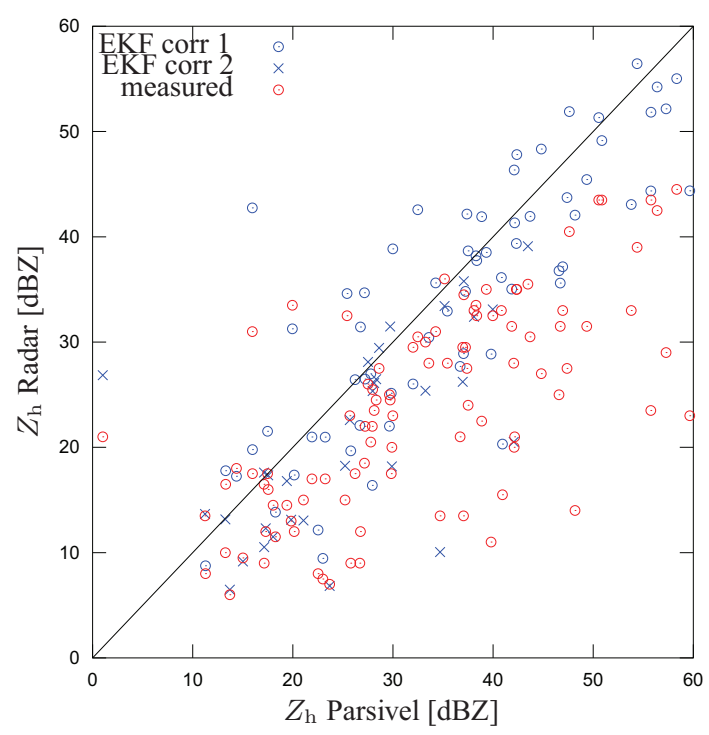

Fig. 14. Scatter plot of the reflectivity $Z_{\mathrm{h}}[\mathrm{dB}]$ inferred from DSD measurements of a Parsivel disdrometer versus radar measurements performed at the range gate and direction corresponding to the location of the disdrometer. The distance between the disdrometer and the radar is $20 \mathrm{~km}$. Red circles show raw (uncorrected) radar measurements; blue circles and crosses show data treated with the EKF algorithm, where the crosses indicate that the offset is determined with the $K_{\mathrm{dp}}$ method instead of the EKF method.

the reflectivity offset associated with high $Z_{\mathrm{h}}$ values is usually determined with the EKF method, since it is likely to have high $\Phi_{\mathrm{dp}}^{\max }$ values during intense precipitation above the radar.

It is seen in the scatter plot that uncorrected reflectivities exhibit a considerable bias and that the attenuation correction (for both the radome and the rain medium) seem to perform well. A statistical analysis of the performance of the correction schemes is given in Table 4, where also the performance of the ZPHI algorithm is evaluated. From this analysis, it is 
Table 4. Standard deviation (std), bias and correlation coefficient between the polarimetric moments calculated from the DSD measurements and the corrected and raw radar measurements. The number of samples is $N=101$.

\begin{tabular}{|c|c|c|c|c|c|c|c|c|c|}
\hline \multirow{2}{*}{ Observable } & \multicolumn{3}{|c|}{ Bias } & \multicolumn{3}{|c|}{ std } & \multicolumn{3}{|c|}{ Correlation } \\
\hline & EKF & ZPHI & raw & EKF & ZPHI & raw & EKF & ZPHI & raw \\
\hline$Z_{h}[\mathrm{dBZ}]$ & 1.59 & 1.32 & 8.24 & 9.10 & 8.80 & 10.83 & 0.782 & 0.801 & 0.723 \\
\hline$Z_{\mathrm{dr}}[\mathrm{dB}]$ & 0.072 & 0.040 & 0.307 & 0.610 & 0.730 & 0.848 & 0.723 & 0.377 & 0.257 \\
\hline$K_{\mathrm{dp}}\left[{ }^{\circ} \mathrm{km}^{-1}\right]$ & 0.799 & - & 0.893 & 2.24 & - & 2.38 & 0.760 & - & 0.731 \\
\hline
\end{tabular}

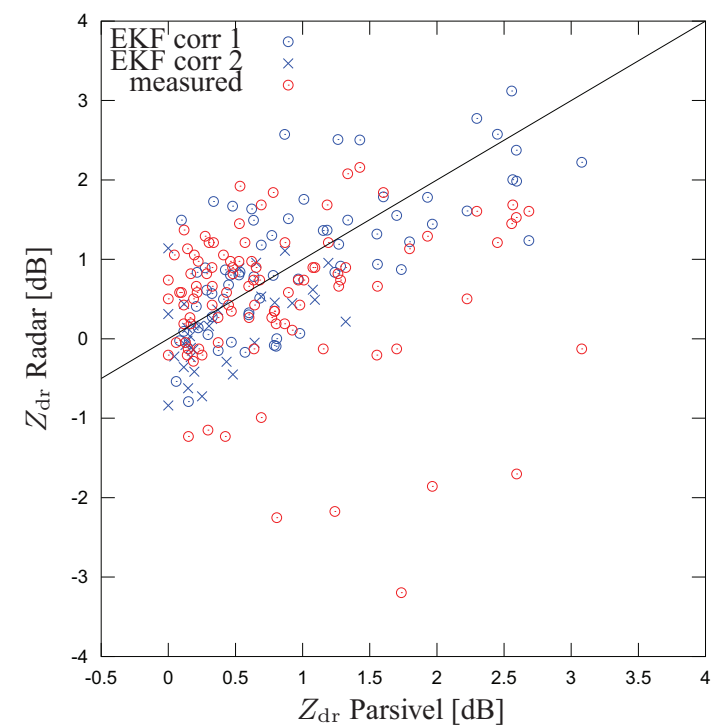

Fig. 15. Same as in Fig. 14 but for $Z_{\mathrm{dr}}$.

concluded that the ZPHI method, applied on radome attenuation corrected data, leads to the most stable reflectivity estimates with a reflectivity bias of $\Delta_{Z_{\mathrm{h}}}=1.32 \mathrm{~dB}$ and a standard deviation of $\sigma_{Z_{\mathrm{h}}}=8.80 \mathrm{~dB}$. Attenuation correction with the EKF algorithm is slightly less accurate $\left(\Delta_{Z_{\mathrm{h}}}=1.59 \mathrm{~dB}\right.$, $\sigma_{Z_{\mathrm{h}}}=9.10 \mathrm{~dB}$ ). The fact that ZPHI performs slightly better is probably due the fact that the accuracy of the rain attenuation correction with ZPHI is not reduced when the radome attenuation is not exactly determined (i.e. when the radar is miscalibrated). Given the large bias of the completely uncorrected data $\left(\Delta_{Z_{\mathrm{h}}}=8.24 \mathrm{~dB}\right)$ and regarding other effects like the spatial variability of the DSD within a radar resolution volume (Jaffrain et al., 2011), the performance of both ZPHI and EKF lead to an acceptable residual bias. The standard deviation of both ZPHI and EKF appears high, but it is a reasonable increase compared to the standard deviation that was found for dry radome conditions (Fig. 11).

Figure 15 shows the same results as Fig. 14 but for the differential reflectivity $Z_{\mathrm{dr}}$. The offset in $Z_{\mathrm{dr}}$ determined in Sect. 6 was added to the measured $Z_{\mathrm{dr}}$ values before the data were further treated with the EKF algorithm and the ZPHI method. Similar to the findings for $Z_{\mathrm{h}}$, it is seen that uncorrected $Z_{\mathrm{dr}}$ data can exhibit a large error and that the

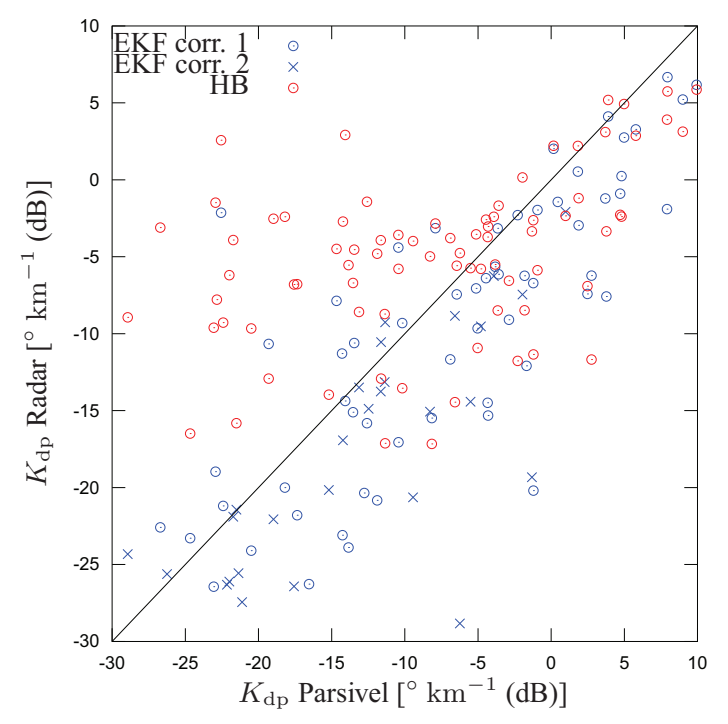

Fig. 16. Same as in Fig. 14 but for $K_{\mathrm{dp}}$. Note that $K_{\mathrm{dp}}$ is not directly measured with a radar, hence the EKF data is compared to $K_{\text {dp }}$ data inferred with the algorithm of Hubbert and Bringi (1995).

attenuation correction with the EKF method performs reasonably. Again, the statistical evaluation is found in Table 4.

While the bias in $Z_{\mathrm{dr}}$ is similar for EKF and ZPHI $\left(\Delta_{Z_{\mathrm{dr}}} \approx 0.05 \mathrm{~dB}\right)$, a slightly better standard deviation is found for $\operatorname{EKF}\left(\sigma_{Z_{\mathrm{dr}}}=0.61 \mathrm{~dB}\right)$ than for ZPHI $\left(\sigma_{Z_{\mathrm{dr}}}=0.73 \mathrm{~dB}\right)$. The reason is found in Fig. 7, where it can be seen that $Z_{\mathrm{dr}}$ data treated with the EKF algorithm are less noisy than the raw or ZPHI treated data. Apparently, this noise reduction not only has a positive effect on $\sigma_{Z_{\mathrm{dr}}}$, but also the correlation coefficient $R_{Z_{\mathrm{dr}}}^{2}$ between the disdrometer and the radar values is distinctly enhanced compared to ZPHI or raw data.

Finally, the same analysis as for $Z_{\mathrm{dr}}$ and $Z_{\mathrm{h}}$ was performed for $K_{\mathrm{dp}}$ and the result is shown in Fig. 16. Since $K_{\mathrm{dp}}$ is a quantity that is not directly measured, the values inferred from the EKF method were compared to data deduced with the algorithm of Hubbert and Bringi (1995), which is based on a smoothing procedure and a subsequent derivation of the measured differential phase $\Psi_{\mathrm{dp}}$. This algorithm was already implemented in the radar's data analysis software and the data were taken as provided without further change. In conditions of high $K_{\mathrm{dp}}$, the EKF algorithm seems to provide 
Table A1. Power law coefficients that are used to correct the EKF parameters for elevations angles $\Theta \neq 0$.

\begin{tabular}{lllllllll}
\hline & $\mu_{\mathrm{h}}$ & $\mu_{\mathrm{V}}$ & $\kappa_{\mathrm{h}}$ & $\kappa_{\mathrm{V}}$ & $\lambda_{\mathrm{h}}$ & $\lambda_{\mathrm{V}}$ & $\zeta$ & $\eta$ \\
\hline$f$ & $1.40 \times 10^{-4}$ & $1.21 \times 10^{-4}$ & $2.86 \times 10^{-3}$ & $2.94 \times 10^{-3}$ & $2.19 \times 10^{-5}$ & $5.29 \times 10^{-5}$ & $4.90 \times 10^{-5}$ & $1.86 \times 10^{-3}$ \\
$g$ & 1.77 & 1.80 & 1.85 & 1.90 & 1.72 & 1.89 & 2.19 & 0.97 \\
\hline
\end{tabular}

slightly underestimated values, while in conditions of low $K_{\mathrm{dp}}$, the algorithm of Hubbert and Bringi (1995) provides too high $K_{\text {dp }}$ estimates. Overall it is seen in Table 4 that $K_{\mathrm{dp}}$ estimated with EKF outperform the traditional $K_{\mathrm{dp}}$ estimates inferred from the algorithm of Hubbert and Bringi (1995) in terms of bias, standard deviation and correlation.

\section{Conclusions}

Polarimetric X-band radar data were collected in in Fortaleza, northern Brazil. It was found that the reflectivity is heavily affected by attenuation of the radome and the rain medium itself. An extended Kalman filter algorithm capable of radome and rain attenuation correction was implemented and data from one month was processed. The radome attenuation in azimuth and elevation direction was not found to be constant within one scan, hence it is recommended to correct every direction independently. In addition to the EKF algorithm, a standard rain attenuation correction scheme (ZPHI) was implemented. The necessary adaptations of this scheme, i.e. the proper calculation of attenuation parameters, was performed with modeled polarimetric observables that are based on DSDs measured with three Parsivel disdrometers. The corrected X-band radar measurements were compared with the disdrometer measurement located in $20 \mathrm{~km}$ distance from the radar. This comparison showed that the implemented algorithms performed satisfactory: Reflectivities corrected with ZPHI exhibited the smallest bias and standard deviation compared to the disdrometer data as well as the highest correlation, the EKF algorithm outperformed ZPHI in the correction of the differential reflectivities. The EKF method was also found to give better estimates of $K_{\mathrm{dp}}$ than the algorithm of Hubbert and Bringi (1995). Given the large biases of uncorrected data, it must be emphasized that without radome attenuation correction such accurate estimates would not have been possible. It was also shown that the variability of the reflectivity offset in direction and time is extremely variable, hence it is strongly recommended to implement a radome attenuation correction scheme that individually corrects for every direction and at every time step. The correction of rain attenuation at X-band frequencies by employing polarimetric techniques is meanwhile a standard procedure. The results of this study suggest that also the correction of radome attenuation should become part of a standard X-band weather radar data processing scheme.
Table B1. Power law coefficients that are used to determine the EKF parameters as a function of the temperature.

\begin{tabular}{rrrrrrr}
\hline & $\mu_{\mathrm{h}}$ & $\mu_{\mathrm{v}}$ & $\kappa_{\mathrm{h}}$ & $\kappa_{\mathrm{V}}$ & $\zeta$ & $\eta$ \\
\hline$k$ & 9.6378 & 4.3133 & 28.2 & 26.175 & 17.726 & 3.77 \\
$l$ & -0.66944 & -0.56760 & 0.077 & 0.083635 & -0.57419 & -0.149 \\
\hline
\end{tabular}

\section{Appendix A}

\section{Elevation angle correction for EKF parameters}

The parameters for the EKF algorithm given in Table 2 were calculated for an elevation angle of $\Theta=0^{\circ}$ and are therefore denoted as $P_{0}^{\mathrm{EKF}}$. For higher elevation angles, these parameters are subject to a slight change, which might influence the performance of the EKF retrieval. In order to mitigate these possible errors, the EKF parameters were calculated for elevation angles of $0^{\circ} \leq \Theta \leq 45^{\circ}$ with a $5^{\circ}$ interval. From these calculations, correction functions of the form

$P_{\Theta}^{\mathrm{EKF}}=P_{0}^{\mathrm{EKF}}+f \Theta^{g}$

were estimated, where $\Theta$ is in [deg] and $f$ and $g$ are power law coefficients that were determined from linear least square fits on the logarithm of $\Theta$ and the logarithm of $P_{\Theta}^{\mathrm{EKF}}-P_{0}^{\mathrm{EKF}}$. The coefficients $f$ and $g$ are given in Table A1 for every EKF parameter.

\section{Appendix B}

\section{Temperature correction for EKF parameters}

Similar to the treatment in the foregoing section of the Appendix, the EKF parameters can also be corrected for different environmental temperatures. The temperature has an effect on the dielectric constant of water and hence influences the scattering amplitudes of the rain drops. The ansatz for the power law that determines the EKF parameter $P^{\mathrm{EKF}}(T)$ as a function of the temperature $T$ is the following:

$P^{\mathrm{EKF}}(T)=k \cdot T^{l}$.

In the above equation, the temperature needs to be given in $\mathrm{K}$. T-matrix calculations were made for a temperature range of $0^{\circ} \mathrm{C}<T<40^{\circ} \mathrm{C}$ with an interval of $1{ }^{\circ} \mathrm{C}$. The power law coefficients $l$ and $k$ were determined similarly to the procedure detailed in Appendix A. The coefficients $l$ and $k$ are 
given in Table B1. It was found that the parameters $\lambda_{\mathrm{h}}$ and $\lambda_{\mathrm{v}}$ only slightly depend on the temperature, hence they are assumed to be constant and no power law coefficients are given. With Eq. (B1) and the coefficients of Table B1, the EKF parameters are calculated for an elevation angle of $0^{\circ}$. If other elevation angles are required, the parameters calculated with Eq. (B1) need to be further corrected with the procedure given in Appendix A.

Acknowledgements. This work is supported by FAPESP grant No. 2009/15235-8. Without the expertise of Cesar de Mello in deploying the radar and making it work as well as the effort of Jorge Luiz Marton, Jorge Mello and Jorge Nogueira in setting up the various other instruments, this campaign would not have taken place. We also thank Meiry Sayuri Sakamoto for hosting us at FUNCEME and for her great support in conducting the campaign in Fortaleza.

Edited by: F. S. Marzano

\section{References}

Anagnostou, E. N., Anagnostou, M. N., Krajewski, W. F., Kruger, A., and Miriovsky, B. J.: High-resolution rainfall estimation from X-band polarimetric radar measurements, J. Hydrometeorol., 5, 110-128, 2004.

Anagnostou, E. N., Grecu, M., and Anagnostou, M. N.: X-band polarimetric radar rainfall measurements in Keys area microphysics project, J. Atmos. Sci., 63, 187-203, 2006.

Andsager, K., Beard, K. V., and Laird, N. E.: Laboratory measurements of axis ratios for large raindrops, J. Atmos. Sci., 56, 26732683, 1999.

Barber, P. and Yeh, C.: Scattering of electromagnetic waves by arbitrarily shaped dielectric bodies, Appl. Optics, 14, 2864-2872, 1976.

Battaglia, A., Rusemeyer, E., Tokay, A., Blahak, U., and Simmer, C.: PARSIVEL snow observations: A critical assessment, J. Atmos. Ocean. Tech., 27, 333-344, 2010.

Bechini, R., Baldini, L., Cremonini, R., and Gorgucci, E.: Differential Reflectivity Calibration for Operational Radars, J. Atmos. Ocean. Tech., 25, 1542-1555, 2008.

Bechini, R., Chandrasekar, V., Cremonini, R., and Lim, S.: Radome attenuation at X-band radar operations, in: Proc. 6th European Conf. on Radar in Meteorology and Hydrology: Adv. in Radar Technology, Sibiu, Romania, 2010.

Berne, A., Delrieu, G., and Andrieu, H.: Estimating the vertical structure of intense Mediterranean precipitation using two Xband weather radar systems, J. Atmos. Ocean. Tech., 22, 16561675, 2005.

Brandes, E., Zhang, G., and Vivekanandan, J.: Experiments in rainfall estimation with a polarimetric radar in a subtropical environment, J. Appl. Meteorol., 41, 674-685, 2002.

Bringi, V. N. and Chandrasekar, V.: Polarimetric Doppler Weather Radar: Principles and Applications, Cambridge University Press, 2001.
Bringi, V. N., Keenan, T. D., and Chandrasekar, V.: Correcting CBand radar reflectivity and differential reflectivity data for rain attenuation: A self-consistent method with constraints, IEEE T. Geosci. Remote, 39, 1906-1915, 2001.

Delrieu, G., Serrar, S., Guardo, E., and Creutin, J.-D.: Rain measurement in hilly terrain with X-band weather radar systems: accuracy of path-integrated attenuation estimates derived from mountain return, J. Atmos. Ocean. Tech., 16, 405-415, 1999.

Gorgucci, E., Scarchilli, G., and Chandrasekar, V.: A procedure to calibrate multiparameter weather radar using properties of the rain medium, IEEE T. Geosci. Remote, 37, 269-276, 1999.

Gorgucci, E., Chandrasekar, V., and Baldini, L.: Correction of Xband radar observation for propagation effects based on the selfconsistency principle, J. Atmos. Ocean. Tech., 23, 1668-1681, 2006.

Hubbert, J. and Bringi, V. N.: An iterative filtering technique for the analysis of copolar differential phase and dual-frequency radar measurements, J. Atmos. Ocean. Tech., 12, 643-648, 1995.

Jaffrain, J. and Berne, A.: Experimental Quantification of the Sampling Uncertainty Associated with Measurements from PARSIVEL Disdrometers, J. Hydrometeorol., 12, 352-370, 2011.

Jaffrain, J., Studzinski, A., and Berne, A.: A network of disdrometers to quantify the small-scale variability of the raindrop size distribution, Water Resour. Res., 47, W00H06, doi:10.1029/2010WR009872, 2011.

Matrosov, S. Y.: Evaluating Polarimetric X-Band Radar Rainfall Estimators during HMT, J. Atmos. Ocean. Tech., 27, 122-134, 2010.

McLaughlin, D., Pepyne, D., Chandrasekar, V., Philips, B., Kurose, J., Zink, M., Droegemeier, K., Cruz-Pol, S., Junyent, F., Brotzge, J., Westbrook, D., Bharadwaj, N., Wang, Y., Lyons, E., Hondl, K., Liu, Y., Knapp, E., Xue, M., Hopf, A., Kloesel, K., Defonzo, A., Kollias, P., Brewster, K., Contreras, R., Dolan, B., Djaferis, T., Insanic, E., Frasier, S., and Carr, F.: Short-Wavelength Technology and the Potential For Distributed Networks of Small Radar Systems, B. Am. Meteorol. Soc., 90, 1797-1817, 2009.

Meissner, T. and Wentz, F. J.: The complex dielectric constant of pure and sea water from microwave satellite observations, IEEE T. Geosci. Remote, 42, 1836-1849, 2004.

Mishchenko, M. I. and Travis, L. D.: Capabilities and limitations of a current FORTRAN implementation of the T-matrix method for randomly oriented, rotationally symmetric scatterers, J. Quant. Spectrosc. Ra., 60, 309-324, 1998.

Park, S. G., Maki, M., Iwanami, K., Bringi, V. N., and Chandrasekar, V.: Correction of radar reflectivity and differential reflectivity for rain attenuation at X-band, Part II: Evaluation and application, J. Atmos. Ocean. Techn., 22, 1633-1655, 2005.

Peters, G., Fischer, B., and Andersson, T.: Rain observations with a vertically looking Micro Rain Radar (MRR), Boreal Environ. Res., 7, 353-362, 2002.

Ryzhkov, V. R., Giangrande, S. E., Melnikov, V. M., and Schuur, T. J.: Calibration issues of dual-polarization radar measurements, J. Atmos. Ocean. Tech., 22, 1138-1155, 2005.

Schleiss, M., Berne, A., and Uijlenhoet, R.: Geostatistical simulation of 2D fields of raindrop size distributions at the meso- $\gamma$ scale, Water Resour. Res., 45, W07415, doi:10.1029/2008WR007545, 2009. 
Schneebeli, M. and Berne, A.: An extended Kalman filter framework for polarimetric X-Band weather radar data processing, J. Atmos. Ocean. Tech., 29, 711-730, 2012.

Schneebeli, M., Berne, A., Jolivet, S., Muth, X., Jaffrain, J., Dawes, N., and Lehning, M.: Measurements of Alpine Precipitation with an X-Band Polarimetric Radar and Additional Sensors, in: Proc. 6th European Conf. on Radar in Meteorology and Hydrology: Adv. in Radar Technology, Sibiu, Romania, 2010.

Seliga, T. A., Bringi, V. N., and Al-Khatib, H. H.: Differential reflectivity measurements in rain: First experiments, IEEE T. Geosci. Elect., 17, 240-244, 1979.

Testud, J., Bouar, E. L., Obligis, E., and Ali-Mehenni, M.: The rain profiling algorithm applied to polarimetric weather radar data, J. Atmos. Ocean. Tech., 17, 332-356, 2000.

Thurai, M. and Bringi, V. N.: Drop axis ratios from a 2D video disdrometer, J. Atmos. Ocean. Tech., 22, 966-978, doi:10.1175/JTECH1767.1, 2005.
Wang, Y. and Chandrasekar, V.: Polarization Isolation Requirements for Linear Dual-Polarization Weather Radar in Simultaneous Transmission Mode of Operation, IEEE T. Geosci. Remote, 44, 2019-2028, 2006.

Wang, Y. and Chandrasekar, V.: Quantitative Precipitation Estimation in the CASA X-band Dual-Polarization Radar Network, J. Atmos. Ocean. Tech., 27, 1665-1676, 2010.

Ware, R., Solheim, F., Carpenter, R., Güldner, J., Liljegren, J. T. N., and Vandenberghe, F.: A multi-channel radiometric profiler of temperature, humidity and cloud liquid, Radio Sci., 38, 8079, doi:10.1029/2002RS002856, 2003.

Zrnic, D. S. and Ryzhkov, A. V.: Polarimetry for weather surveillance radars, B. Am. Meteorol. Soc., 80, 389-406, 1999. 